\title{
Activation of murine pre-proglucagon-producing neurons reduces food intake and body weight
}

\author{
Ronald P. Gaykema, ${ }^{1}$ Brandon A. Newmyer, ${ }^{1}$ Matteo Ottolini, ${ }^{2}$ Vidisha Raje, ${ }^{1}$ Daniel M. Warthen, ${ }^{1}$ Philip S. Lambeth, \\ Maria Niccum, ${ }^{1}$ Ting Yao, ${ }^{3,4}$ Yiru Huang, ${ }^{4,5}$ Ira G. Schulman, ${ }^{1}$ Thurl E. Harris, ${ }^{1}$ Manoj K. Patel, ${ }^{2}$ \\ Kevin W. Williams, ${ }^{4}$ and Michael M. Scott ${ }^{1}$ \\ 'Department of Pharmacology, ${ }^{2}$ Department of Anesthesiology, University of Virginia School of Medicine, Charlottesville, Virginia, USA. ${ }^{3}$ Department of Physiology and Pathophysiology, \\ Xi'an Jiaotong University School of Medicine, Xi'an, China. 'Division of Hypothalamic Research, Department of Internal Medicine, University of Texas Southwestern Medical Center, \\ Dallas, Texas, USA. ${ }^{5}$ Department of Neurosurgery, Zhujiang Hospital, Southern Medical University, Guangzhou, China.
}

Peptides derived from pre-proglucagon (CCC peptides) act in both the periphery and the CNS to change food intake, glucose homeostasis, and metabolic rate while playing a role in anxiety behaviors and physiological responses to stress. Although the actions of GCC peptides produced in the gut and pancreas are well described, the role of glutamatergic GCC peptide-secreting hindbrain neurons in regulating metabolic homeostasis has not been investigated. Here, we have shown that chemogenetic stimulation of GCG-producing neurons reduces metabolic rate and food intake in fed and fasted states and suppresses glucose production without an effect on glucose uptake. Stimulation of CCG neurons had no effect on corticosterone secretion, body weight, or conditioned taste aversion. In the diet-induced obese state, the effects of GCG neuronal stimulation on gluconeogenesis were lost, while the food intake-lowering effects remained, resulting in reductions in body weight and adiposity. Our work suggests that CCC peptide-expressing neurons can alter feeding, metabolic rate, and glucose production independent of their effects on hypothalamic pituitary-adrenal (HPA) axis activation, aversive conditioning, or insulin secretion. We conclude that GCG neurons likely stimulate separate populations of downstream cells to produce a change in food intake and glucose homeostasis and that these effects depend on the metabolic state of the animal.

\section{Introduction}

The pre-proglucagon $(G c g)$ gene product peptides glucagon-like peptide 1 (GLP-1), GLP-2, oxyntomodulin (OXM), intervening peptide 1 (IP1), and glicentin (hereafter referred to as GCG peptides), produced through the actions of prohormone convertase $1 / 3$ (reviewed in ref. 1), are released by a small population of L cells in the intestine, altering metabolic homeostasis, insulin secretion (2-5), and food intake (6).

Interestingly, these peptides are also released by a small population of neurons in the caudal hindbrain of both rodents and humans (7-12) and likely act in the brain to change animal physiology.

For example, injection of GLP-1 or GLP-1 receptor (GLP-1R) agonists (exendin-4 or liraglutide) into various brain regions - including the lateral ventricles (7) or parenchyma of the rodent lateral (13) and arcuate (14) divisions of the hypothalamus, the nucleus accumbens core (15), lateral parabrachial nucleus (16), and nucleus of the solitary tract (17) - robustly reduces food intake. Furthermore, GLP-1 has also been shown to induce pica (18) and conditioned taste aversion (19), through action at GLP-1Rs expressed at specific neuroanatomical sites that are distinct from those regulating satiety (20-23).

\section{Related Commentary: p. 793}

Authorship note: K.W. Williams and M.M. Scott contributed equally to this work Conflict of interest: The authors declare that no conflict of interest exists. Submitted: February 3, 2015; Accepted: December 30, 2016. Reference information: / Clin Invest. 2017;127(3):1031-1045. https://doi.org/10.1172/JCl81335
The central actions of GLP-1 on glucose homeostasis, in contrast to the effects on food intake, appear to be more complex. In particular, GLP-1R is not required in the central nervous system to maintain normal glucose homeostasis (24). These data contrast, however, with work showing that injection of GLP-1 into the arcuate nucleus is sufficient to produce a reduction in liver glucose production (25), while central GLP-1 reduces regulation of glucose uptake (26). GLP-2 receptor deletion from arcuate neurons, meanwhile, impairs the suppression of liver gluconeogenesis, with intracerebroventricular (ICV) infusion of GLP-2 improving glucose tolerance and insulin sensitivity (27). Furthermore, the acute actions of GLP-1 in the regulation of glucose homeostasis may also involve a brief increase in circulating glucose levels brought about by an enhancement in sympathetic outflow $(28,29)$.

Finally, GLP-1 also stimulates the hypothalamic-pituitaryadrenal (HPA) axis (30-33), producing an increase in adrenocorticotropic hormone (ACTH) and corticosterone secretion, through actions in the paraventricular nucleus. Enhancement of anxiety-like behavior through actions at the central nucleus of the amygdala (CeA) (34) has also been observed, while stress-dependent anorexia requires GLP-1R-mediated signaling (35).

Although these pharmacological and receptor deletion studies show how central GLP receptors can produce important effects on physiology and behavior, the contribution by GCG-producing neurons (GCG neurons) has yet to be examined. Gaining a better understanding of how the GCG system functions in the CNS is extremely important, as drugs that target GLP-1R are used clinically as both weight loss and antidiabetic glucose-lowering ther- 
apies (as reviewed in ref. 36). Whether all of the effects observed following pharmacological activation of the GLP-1R can be elicited by GCG neuron stimulation has not been investigated. Clearly, while studies have been able to pharmacologically establish the function of the central GLP-1 system, it is unknown whether intraparenchymal or ICV delivery of GLP-1 or GLP-1 mimetics recapitulates the effect of endogenous neuronal GLP-1 signaling. Furthermore, agonism of GLP-1R cannot be expected to produce the same effects on postsynaptic neurons when compared with stimulation of the GCG neuron itself, which also releases glutamate (11) along with GLP-2, OXM, IP1, and glicentin. It is therefore possible that targeting GCG neurons may present as an even more efficacious therapy in the treatment of obesity and diabetes than simply targeting the individual GCG peptide receptors themselves.

To develop a clearer understanding of GCG neuron function, we have developed a transgenic mouse line expressing Cre recombinase from the $G c g$ locus of a bacterial artificial chromosome (BAC). Through the expression of a designer receptor exclusively activated by designer drugs (DREADD) $(37,38)$, we selectively activated hindbrain GCG neurons using the synthetic ligand clozapine $\mathrm{N}$-oxide (CNO). Surprisingly, we observed that stimulation recapitulated only a subset of the actions ascribed to centrally administered GCG peptides, reducing food intake, metabolic rate, and glucose production in lean animals. No effect was observed on corticosterone secretion, anxiety-like behavior, conditioned taste aversion, or body weight homeostasis. Furthermore, in diet-induced obese (DIO) mice, the effect of GCG neuronal stimulation on glucose production was lost, while the food intake-lowering effect remained, resulting in a reduction of body weight and adiposity.

\section{Results}

Cre recombinase is exclusively expressed in GCG neurons. Gcg-Cre mice were crossed with tdTomato reporter mice to characterize the expression pattern of the transgene (Figure 1A). The presence of tdTomato was detected directly by epifluorescence, and colocalization in GCG neurons was determined by concurrent immunohistochemical detection of GLP-1 peptide. Extensive colocalization of GLP-1 and tdTomato fluorescence was present in the caudal nucleus tractus solitarius (NTS) and ventrolateral medulla (VLM) (Figure 1, D and G). Specificity of the Cre reporter gene was high, with $99.1 \% \pm 0.2 \%$ of tdTomato-labeled cells displaying GLP-1 IR in the NTS, while $91.3 \% \pm 3.1 \%$ of those in the VLM showed coexpression (Figure 1H). The efficacy of Cre recombinase expression in GCG neurons was also high, with $92.6 \% \pm 0.8 \%$ and $83.9 \% \pm 1.1 \%$ of GLP-1-labeled neurons harboring tdTomato in the NTS and VLM, respectively (Figure 1I). Dense axonal plexus and terminal labeling in GCG neuronal target areas such as the paraventricular (Figure 1J) and arcuate (Figure 1K) nuclei of the hypothalamus was also observed. Very limited off-target expression (1-2 neurons per 40- $\mu \mathrm{m}$ section) was seen throughout several brain nuclei, including the olfactory bulb, anterior olfactory nucleus, piriform cortex, medial and cortical amygdala, posterior hypothalamic and ventral premammillary nuclei, and ventral periaqueductal gray/dorsal raphe region (data not shown). The $G c g$ Cre mice exhibited normal body weight regulation (Figure $1 \mathrm{~L}$ ), average daily food intake (Figure $1 \mathrm{M}$ ), and blood glucose levels in both fed and fasted states (Figure 1, N and O).
Expression of $h M 3 D q$ in medullary GCG neurons and chemogenetic activation by the synthetic ligand CNO. AAV5-hSyn-DIO-hM3Dq-mCherry, which encodes an mCherry-tagged Gq-coupled DREADD receptor (hereafter referred to as Gq DREADD), was delivered into the caudal medulla (Figure 2A) to drive the activation of GCG neurons, with experiments conducted 3 weeks after surgery. Following experimentation, mice were injected with $\mathrm{CNO}$, food was removed from all cages, and mice were transcardially perfused 2 hours later to assess viral expression of mCherry and activation by detection of Fos expression.

mCherry (visualized using a dsRed antibody) was seen almost exclusively in neurons that exhibited GLP-1 staining in the caudal NTS (Figure 2B) and VLM (Figure 2C). dsRed neurons displayed Fos immunoreactivity (IR) (intense black cellular nuclei) throughout the caudal NTS (Figure 2D) and VLM (Figure 2E) after CNO treatment. $98.0 \% \pm 0.6 \%$ and $90.4 \% \pm 1.2 \%$ of dsRed cells were Fos-positive in the NTS and VLM, respectively (Figure 2F). Furthermore, intense nuclear Fos staining was largely confined to GLP-1-labeled somata in both the NTS $(73.7 \% \pm 4.1 \%)$ and the VLM $(86.5 \% \pm 1.7 \%)$ (Figure 2, G-I). DsRed- and GLP-1-labeled neurons were seen within the medullary raphe pallidus ventral to the hypoglossal motor nucleus (XII) and rostral to the pyramidal decussation and were double labeled for Fos IR (DsRed: 97.6\% $\pm 2.0 \%$, GLP-1: 79.2\% $\pm 4.2 \%$ ). Mice expressing Gq-coupled DREADD receptors in GCG neurons (GCG-Gq DREADD mice) that received saline injections lacked Fos IR within the DsRed-labeled (Figure 2J) and GLP-1-positive cell population (Figure 2K), just like the GCG neurons obtained from WT siblings that underwent the same procedure (data not shown). Figure 2L depicts the characteristic distribution of GCG neurons in the caudal medulla for reference. In the same cohorts, CNO treatment increased Fos IR slightly in two GCG neuron target regions: the paraventricular ( $\mathrm{PVH}, 356 \pm 34$ vs. $218 \pm 21, P=0.015$; Figure 2, $\mathrm{M}-\mathrm{O}$ ) and arcuate hypothalamic nuclei (Arc; $299 \pm 19$ vs. $208 \pm 12$, $P=0.005$; Figure 2, $\mathrm{P}-\mathrm{R})$.

Electrophysiology. Expression of the virally introduced Gq DREADD receptor was confirmed by visual inspection of fluorescent neurons in tissue slices (Figure 3A) and targeted for whole cell patch clamp recordings (Figure 3B). While low concentrations of CNO (1 and $3 \mu \mathrm{M}$; data not shown) failed to increase GCG neuronal firing, 9 $\mu \mathrm{M}$ did produce a small increase in action potential generation (Figure 3, C and D). Increases in CNO concentration from $9 \mu \mathrm{M}$ to 12 $\mu \mathrm{M}$ did not produce further neuronal potentiation (data not shown). This suggested that our in vivo dosing produces an effect on physiology that recapitulates the effect of $9 \mu \mathrm{M}$ CNO in vitro. Bath application of $\mathrm{CNO}(9 \mu \mathrm{M} ; 10$ minutes) evoked depolarizing events that triggered few action potentials solely in mCherry-labeled neurons ( $n=6 \pm 0.4$ events in 10 minutes; Figure 3, C and D) and produced an elevation in resting membrane potential (example in Figure 3E; 6-mV change). CNO had no effect on unlabeled neurons (Figure 3, $\mathrm{F}$ and $\mathrm{G} ; n=5$ from 4 animals). Next, we investigated whether CNO can potentiate action potential generation following current injection. Prior to bath application of CNO, injection of depolarizing current steps induced action potential firing in mCherry-labeled and unlabeled neurons (Figure 3, $\mathrm{H}$ and $\mathrm{K}$ ). Superfusion of $9 \mu \mathrm{M} \mathrm{CNO}$ produced a 2-fold potentiation of neuronal firing frequency (before $\mathrm{CNO}, 7.7 \pm 2.0 \mathrm{~Hz}$ and in $\mathrm{CNO}, 14.3 \pm 2.0 \mathrm{~Hz}$; 2-way repeated measures ANOVA, $F_{1,10}=7.782, P=0.019$; Figure 3, I and J). Notably, this 
A
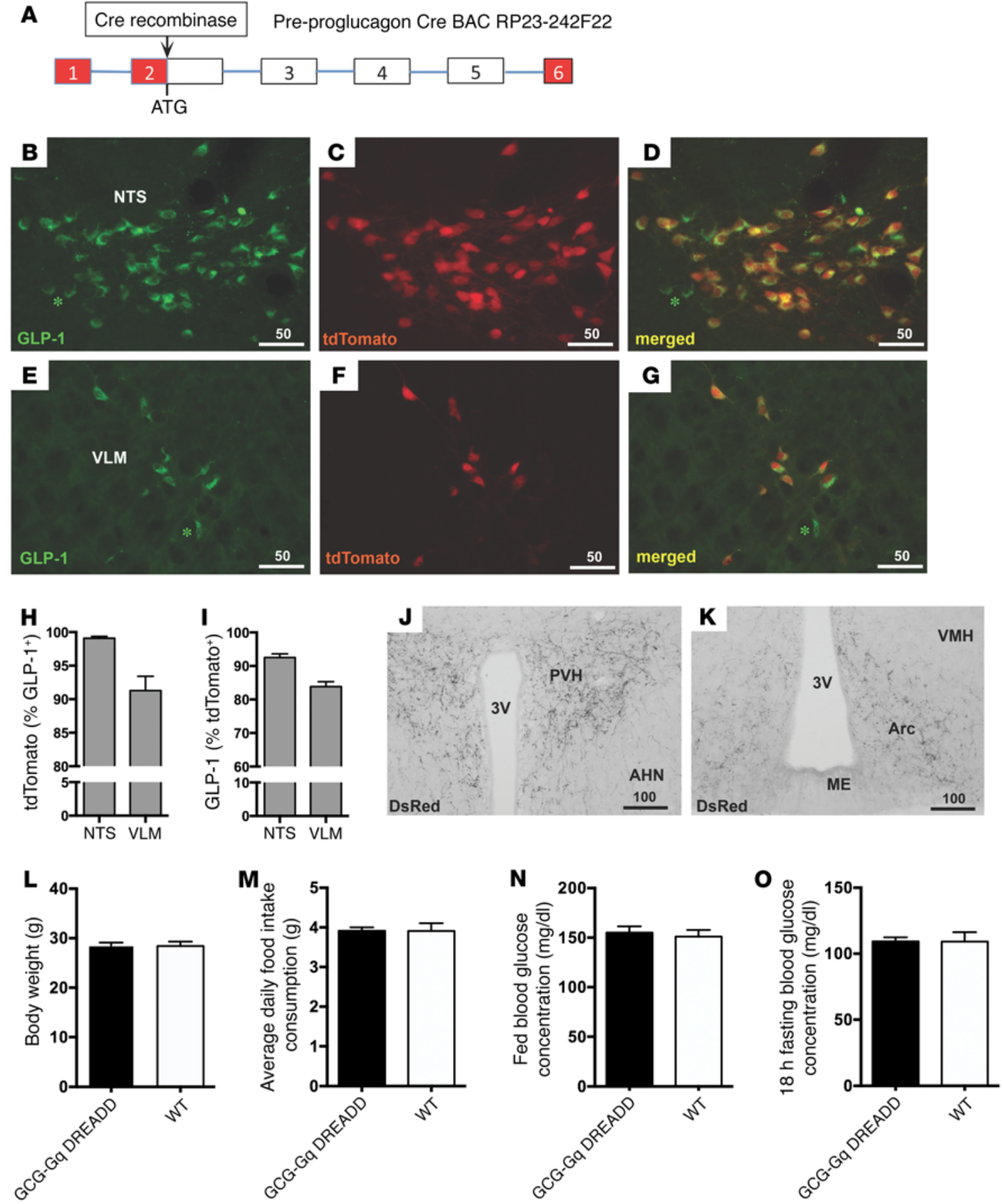

Figure 1. Characterization of $\mathbf{G c g}$-Cre mice. (A) Cre recombinase was inserted into the Gcg gene locus of BAC RP23-242F22 at the ATC start codon. Red boxes, 5' and 3' untranslated regions; white boxes, Gcg exons; lines, intronic DNA. (B-I) Cre recombinase expression within GCG neurons was visualized by crossing Gcg-Cre mice with tdTomato reporter animals. tdTomato fluorescence (NTS in C; VLM in F) is restricted to GLP-1-expressing cells (B and E) in the NTS (D) and the VLM (G). Specificity of Cre recombinase expression is high, with virtually all tdTomato-labeled cells in the NTS (99\%) and more than 92\% in the VLM showing GLP-1 immunofluorescence (H). The majority of GLP-1-labeled neurons show Cre recombinase activity, with efficacy ranging from $84 \%$ in the VLM to $93 \%$ in the NTS (I). The few GLP-1-positive neurons that lack tdTomato signal are marked with green asterisks (in B, D, E, and $\mathbf{C}$ ). (J and $\mathbf{K}$ ) $\mathrm{PVH}$ and arcuate nuclei (Arc) neurons did not show expression, but tdTomato-labeled axons and terminals originating from brainstem GCG neurons were readily observed. AHE, anterior hypothalamic nucleus; ME, median eminence. (L-O) GCG-Gq DREADD transgenic mice exhibit normal body weight (L), daily chow food intake (M), and normal fed (N) and fasting $(\mathbf{0})$ glucose levels. Scale bars in $\mathbf{B}-\mathbf{G}, \mathbf{J}$, and $\mathbf{K}$ are in micrometers.

was a change similar to that elicited by cholecystokinin (CCK) and leptin, endogenously expressed peptides shown to modulate the activity of GCG neurons $(39,40)$. The action potential firing frequency in unlabeled neurons was not affected by CNO perfusion
(Figure 3M). Together, these data suggest that DREADD stimulation produces a physiologically relevant activation of GCG neurons.

GCG neuronal stimulation in lean mice is sufficient to produce a significant reduction in food intake. Pharmacological activation of 


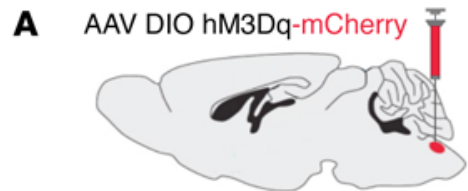

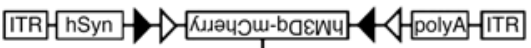
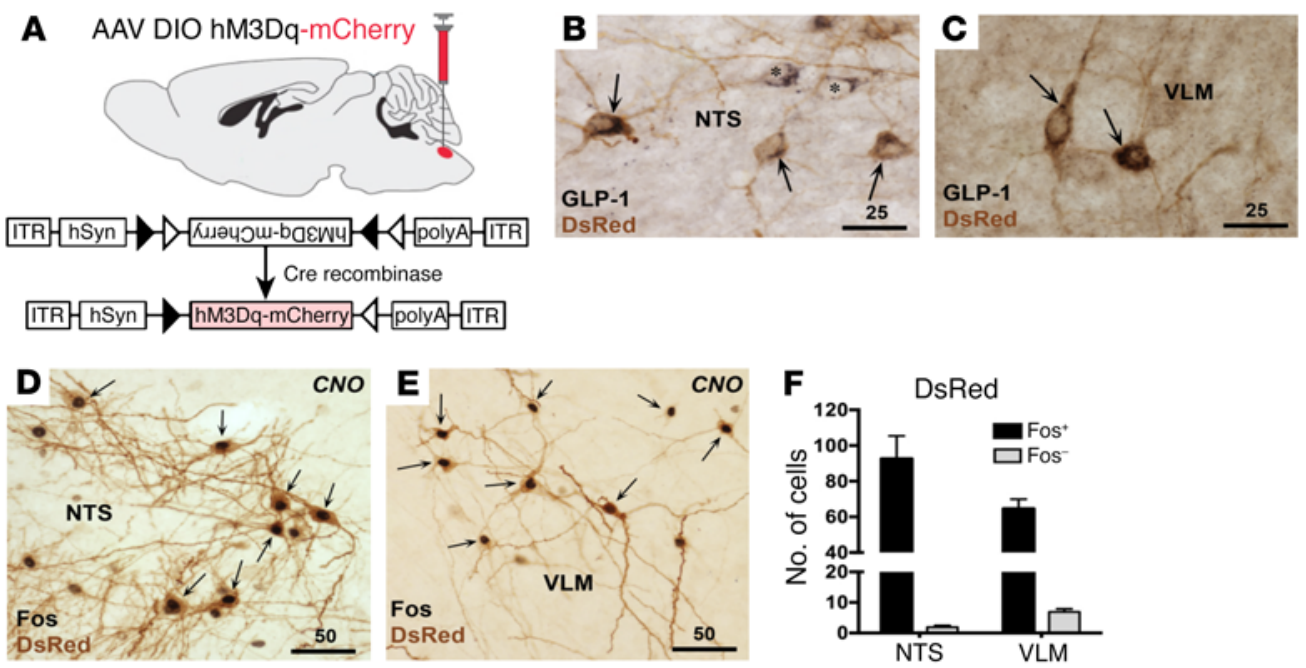

G

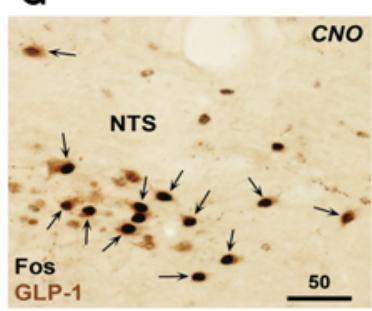

H
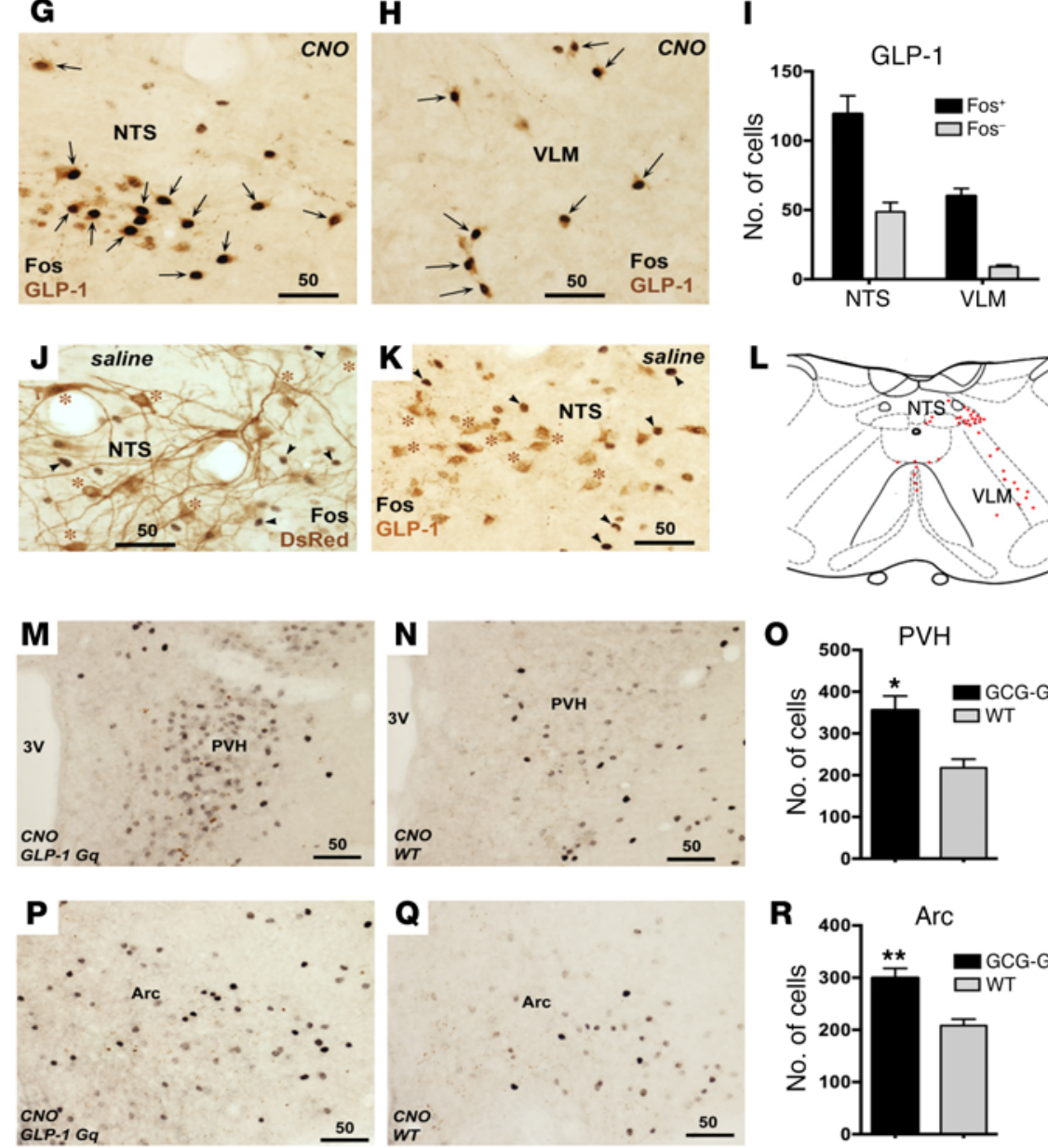
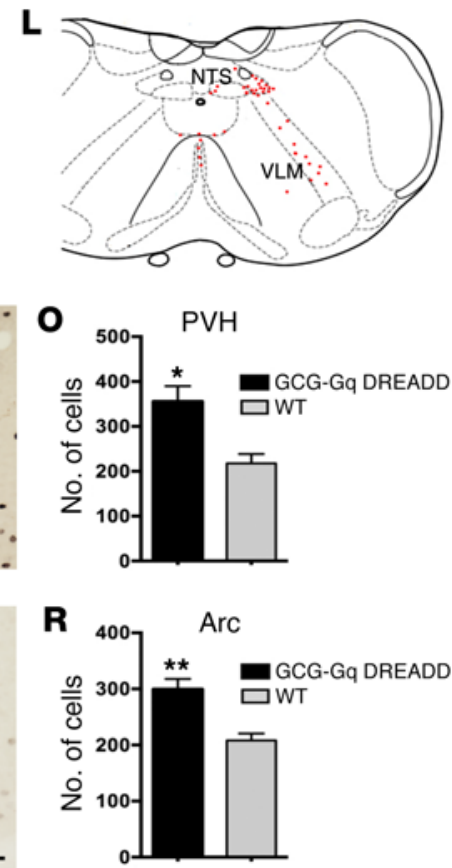

Figure 2. Activation of GCG neurons transfected with Gq DREADD.

Microinjection of AAV2/5-hSyn-DIO$\mathrm{hM}_{3} \mathrm{Dq}(\mathrm{Gq})$ - $\mathrm{mCherry}(\mathbf{A})$ resulted in Gq DREADD expression exclusively in GLP-1-immunoreactive neurons of the NTS (B) and the VLM (C); GLP-1, black precipitate; mCherry, brown precipitate. ITR, inverted terminal repeat. Some GLP-1-labeled cells lacked DsRed labeling (marked with asterisks in B). (D-F) Functional Gq DREADD expression was demonstrated by intense Fos induction within DsRed-labeled neurons of the NTS (D) and VLM (E) 2 hours following CNO injection. Strong Fos IR was seen in DsRed-labeled neurons (F). CNO treatment induced intense Fos staining largely limited to neurons displaying GLP-1 IR in the NTS (G) and the VLM (H). Most GCG neurons showed strong Fos staining in both NTS and VLM (I). Two saline-injected GCG-Gq DREADD mice showed a complete lack of Fos IR within DsRed- (J) and GLP-1-immunolabeled neurons (K). (L) Distribution of GCG neurons in the caudal medulla. (M-R) A small but significant increase in CNO-induced Fos staining was seen in the PVH ( $\mathbf{M}, \mathbf{N}$, and $\mathbf{0})$ and the arcuate nucleus (Arc; $\mathbf{P}$, $\mathbf{Q}$, and $\mathbf{R})$, when GCG-Gq DREADD mice ( $\mathbf{M}$ and $\mathbf{P}$ ) were compared with controls ( $\mathbf{N}$ and $\mathbf{Q}$ ). $n=4$ animals per group; all comparisons made using $t$ test, ${ }^{*} P<0.05,{ }^{* *} P<0.01$. Scale bars are in micrometers. central GLP-1Rs has clearly been shown to lead to a reduction in food intake $(7,15,17,20,26,41-45)$. To examine whether central GCG neuron activation is sufficient to reduce feeding, we stimulated GCG neurons in fed and fasted animals and measured chow food and high-calorie diet (HCD) intake. CNO reduced the amount of chow consumed in the GCG-Gq DREADD group when compared with their food intake after receiving saline injections. When regular chow was removed for 2 hours and returned
1 hour after injection, the GCG-Gq DREADD mice consumed significantly less during daytime hours (Figure 4A; $0.12 \pm 0.03$ vs. $0.28 \pm 0.04 \mathrm{~g}$ chow, $t=2.90, P=0.02$ ) and during the first 3 darkphase hours (Figure $4 \mathrm{~B} ; 1.56 \pm 0.16$ vs. $2.29 \pm 0.26 \mathrm{~g}$ chow, $t=2.27$, $P=0.05)$ when compared with saline injection. CNO also diminished intake of HCD (Figure 4C) (2-way repeated measures ANOVA, significant effect of treatment [CNO vs. saline], $F_{1,12}=$ $8.66, P=0.0134)$. We then tested whether GCG neuronal activa- 

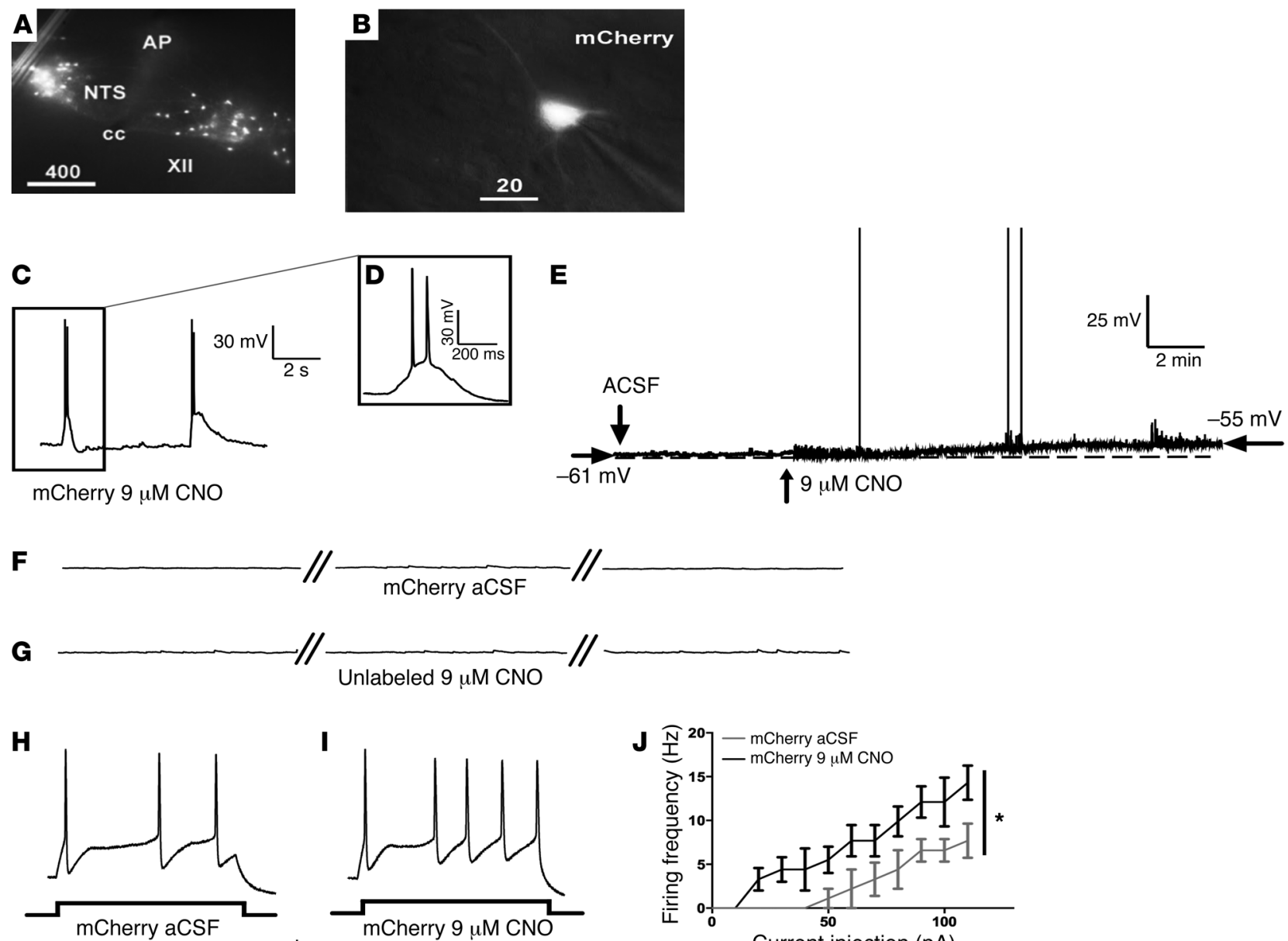
$30 \mathrm{mV} \bigsqcup_{100 \mathrm{~ms}}$

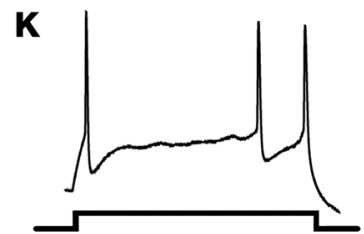

Unlabeled aCSF
I

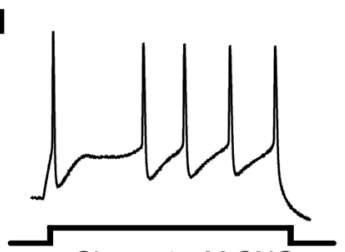

mCherry $9 \mu \mathrm{M}$ CNO

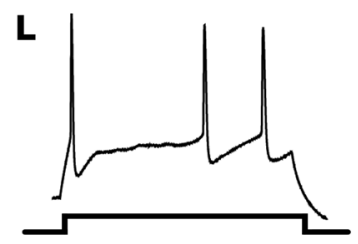

Unlabeled $9 \mu \mathrm{M}$ CNO

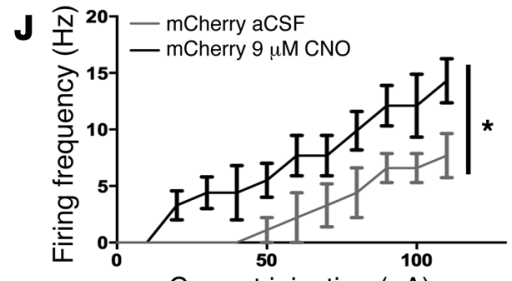

Current injection ( $p A)$
M

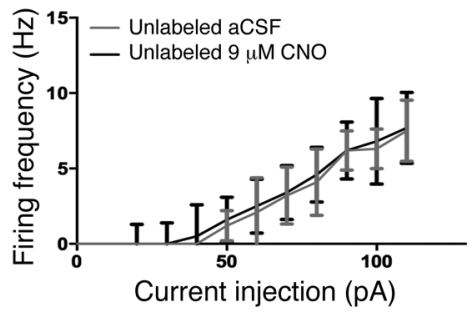

Figure 3. CNO application produces a small increase in GCG neuronal resting membrane potential and increases firing frequency following current injection. CNO elevates resting membrane potential and potentiates action potential firing in mCherry-labeled neurons in the NTS following current injection. Recordings were made from tissue sections from 4 animals. (A) mCherry expression within the NTS. AP, area postrema; cc, central canal. (B) Patch clamped mCherry neuron. (C and D) Representative traces showing that bath application of CNO (9 $\mu \mathrm{M} ; 10$ minutes) evoked firing in mCherry-labeled neurons. (E) An expanded trace showing a single depolarizing event with spikes during perfusion of CNO $(9 \mu \mathrm{M})$. Resting membrane potential was increased by $6 \mathrm{mV}$, an effect that led to a minimal increase in spontaneous firing rate. ACSF, artificial cerebrospinal fluid. (F) Labeled neurons did not fire action potentials in the absence of CNO. (G) CNO $(9 \mu \mathrm{M})$ did not evoke spikes in unlabeled neurons. Breaks shown represent 2 minutes of duration. Spikes elicited by a depolarized current injection step to $110 \mathrm{pA}$ in labeled neurons before $(\mathbf{H})$ and after 10 minutes of CNO $(9 \mu \mathrm{M})$ application (I). (J) Plot of firing frequency as a function of increasing depolarizing current steps shows a significant increase in firing frequency in labeled neurons after 10 minutes of CNO application $(9 \mu \mathrm{M}$; 2-way repeated measures ANOVA, main effect of CNO treatment, $F_{1,10}=7.782, P=0.019$ ). Spikes elicited by a depolarized current injection step to $110 \mathrm{pA}$ in unlabeled neurons before (K) and (L) after 10 minutes of CNO $(9 \mu \mathrm{M})$ application. (M) Plot of firing frequency as a function of increasing depolarizing current steps shows no change in firing frequency in unlabeled neurons after 10 minutes of CNO $(9 \mu \mathrm{M} ; n=5)$. Values represent mean $\pm \operatorname{SEM}$. ${ }^{*} P<0.05$.

tion could reduce food intake following a fast. We hypothesized that fasting-driven food intake could in fact be reduced by GCG neuronal stimulation, even though central GLP-1R modulation has previously been shown to have no effect (46). Indeed, following 18 hours of fasting, consumption of chow during refeeding starting 30 minutes after $\mathrm{CNO} /$ saline injection was significantly reduced (Figure 4D; 2-way repeated measures ANOVA, significant effect of treatment [CNO vs. saline], $F_{1,12}=5.188, P=0.043$ ). To exclude the possibility that $\mathrm{CNO}$ had an effect on feeding via a mechanism other than direct action on the Gq DREADD, a group 

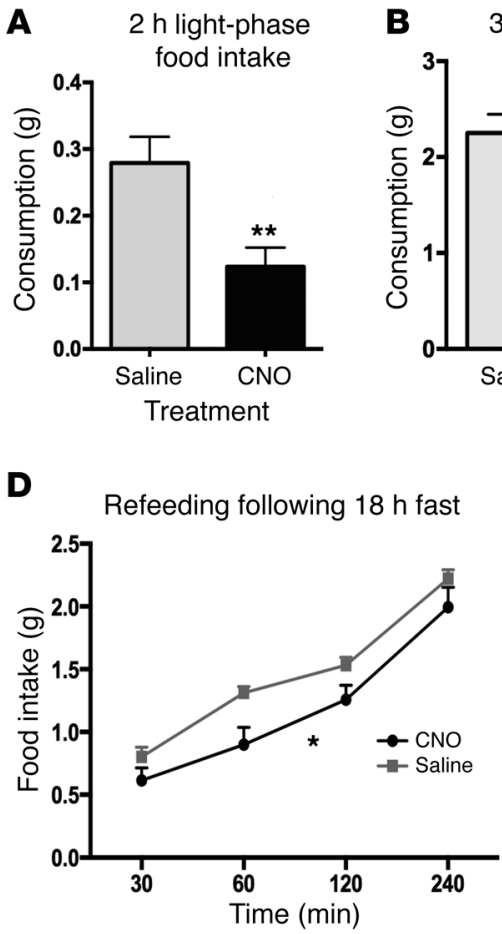
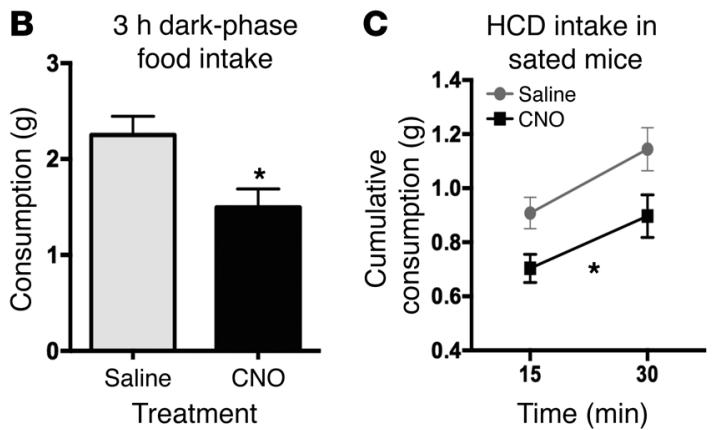

E Refeeding following $18 \mathrm{~h}$ fast WT Gcg-Cre littermates

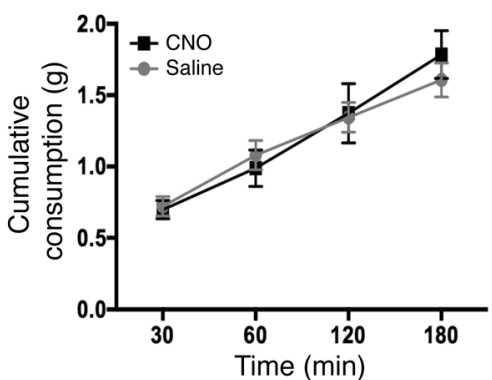

F HCD intake in sated mice

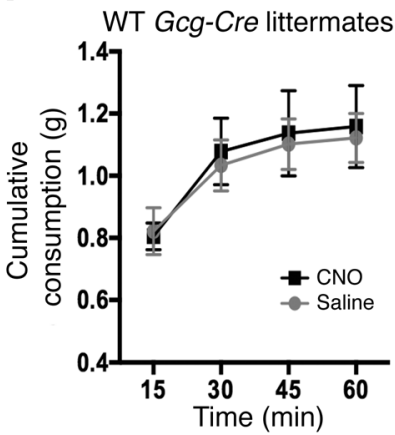

Figure 4. GCG neuron activation modulates food intake in both fed and fasted animals. Activation of GCG neurons by CNO ( $2 \mathrm{mg} / \mathrm{kg})$ reduced food intake both in the light phase (A, paired $t$ test, $n=11,{ }^{* *} P=0.005$ ) and during the first 3 hours of the dark phase (B, paired $t$ test, $\left.n=8,{ }^{*} P=0.026\right)$ upon return of food 2 hours after i.p. CNO/saline injections. HCD intake during early daytime when mice were sated (following ad libitum overnight feeding on chow) was significantly reduced after CNO injection (C, 2-way repeated measures ANOVA, main effect of treatment $F_{111}=8.66,{ }^{*} P=0.0134$ ). Food intake during daytime refeeding following 18 hours of fasting was also significantly reduced (D, 2-way repeated measures ANOVA, $n=13$, main effect of treatment, $\left.F_{1,12}=5.118,{ }^{*} P=0.0430\right)$. WT littermates that underwent sham brain surgery were tested by injection of either CNO (5 mg/kg) or saline i.p., and no effects of CNO were apparent during refeeding after fasting (E, 2-way repeated measures ANOVA, $\left.F_{1,5}=0.06308, P=0.8\right)$ or during HCD feeding $(\mathbf{F}, 2$-way repeated measures ANOVA, $F_{1,5}=0.07558, P=0.62$ ).

of WT (Cre-negative) littermates were subjected to the same feeding protocols, and their food intake was identical after $\mathrm{CNO}$ and saline injections both during refeeding after 18 hours of fasting (Figure 4E; 2-way repeated measures ANOVA, $F_{1,5}=0.0638$, $P=0.8$ ) and when given HCD while satiated (Figure 4F; 2-way repeated measures ANOVA, $F_{1,5}=0.07558, P=0.62$ ).

GCG neuronal stimulation in lean animals has minimal effects on glucose handling and does not affect insulin sensitivity. Central GLP$1 \mathrm{R}$ agonism and antagonism have been shown to improve glucose tolerance, reduce muscle glucose uptake, and reduce liver glucose production $(26,47,48)$. Interestingly, GCG neuronal stimulation did not affect glucose tolerance following CNO administration (Figure 5A; 2-way repeated measures ANOVA, $F_{1,11}=0.04572$, $P=0.8346)$. Insulin sensitivity was also unaffected by CNO treatment (Figure 5B; 2-way repeated measures ANOVA, $F_{1,15}=0.4948$, $P=0.4926)$. On the other hand, ad libitum fed mice showed a small rise in glucose 1 hour after CNO injection compared with a minimal drop in blood glucose levels 1 hour after saline injection, when food was removed for 2 hours prior to glucose measurement (Figure 5C; CNO: $+11.3 \pm 6.5 \mathrm{mg} / \mathrm{dl}$, saline: $-7.3 \pm 4.1 \mathrm{mg} /$ $\mathrm{dl}$; paired $t=2.39, P=0.04)$. These data suggest that activation of the medullary GCG neurons is not sufficient to modulate insulin sensitivity or function, but may affect glucose levels acutely, as described in prior reports (28). We next investigated the effect of GCG neuronal activation on glucose production using a pyruvate tolerance test and glucose uptake using a 2-deoxyglucose uptake assay. While GCG neuronal stimulation reduced gluconeogenesis (Figure 5D; 2-way repeated measures ANOVA, significant effect of treatment [CNO vs. saline], $F_{5,35}=24.24, P=0.001$; Figure $5 \mathrm{E}$, area under the curve, paired $t$ test, $t=2.706, P=0.0304$ ), no effect on glucose uptake was observed (Figure $5 \mathrm{~F}$; $t$ test for each tissue sourced from saline- and CNO-treated animals: extensor digitorum longus [EDL] $t=0.3492, P=0.7388$; tibialis anterior [TA] $t=0.007964, P=0.9939$; soleus $t=0.893, P=0.398$; WAT $t=0.5955, P=0.5732$; liver $t=0.4717, P=0.6538$ ). Finally, fasted insulin levels were shown to be unchanged following CNO treatment (Figure 5G; $t$ test, $n=8, t=0.056, P=0.956$ ).

GCG neuronal stimulation produces a small reduction in energy expenditure but does not affect metabolic fuel preference or body weight in lean animals. The ability of GCG-derived peptides to alter metabolic rate is controversial, with both an increase and decrease in rate suggested to occur following GLP-1R activation (41, 47, 49, $50)$. We tested whether oxygen consumption, $\mathrm{CO}_{2}$ production, and respiratory exchange ratio (RER) were altered in GCG-Gq DREADD mice during the first 3 hours of the dark period after injection of $\mathrm{CNO}$ or saline, during which time food was removed. A small but significant drop after CNO treatment occurred in $\mathrm{O}_{2}$ consumption $\left(\mathrm{VO}_{2}, 3,129 \pm 105\right.$ vs. $\left.3,352 \pm 102 \mathrm{ml} / \mathrm{kg} / \mathrm{h}\right)$ and $\mathrm{CO}_{2}$ production $\left(\mathrm{VCO}_{2}, 2,145 \pm 90\right.$ vs. $2,300 \pm 77 \mathrm{ml} / \mathrm{kg} / \mathrm{h}$ ) (Figure 6A; paired $t$ tests, $t=2.66$ and $2.27, P=0.03$ and 0.05 , respectively), 
A

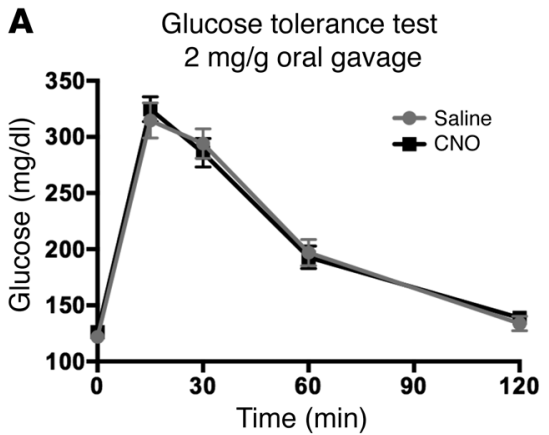

D
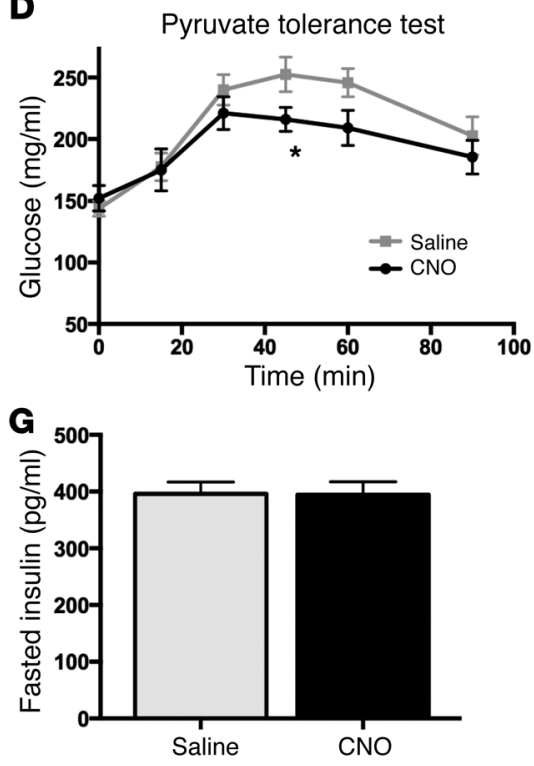

B

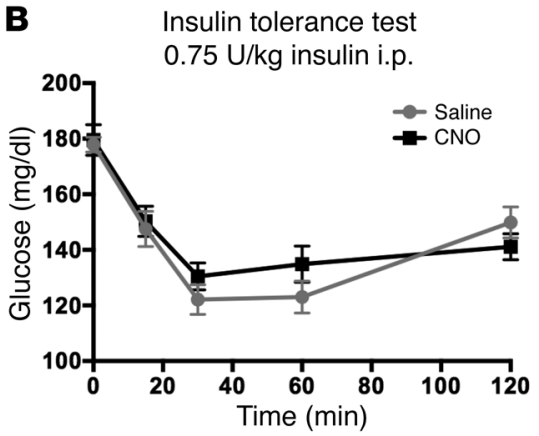

E

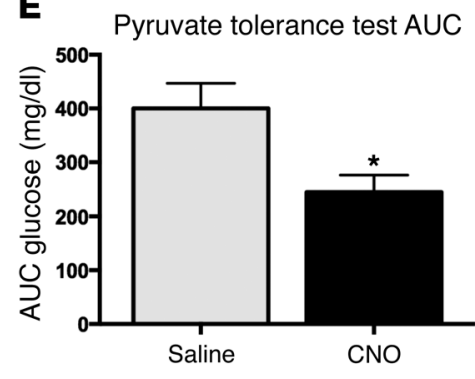

$\mathbf{F}$
C
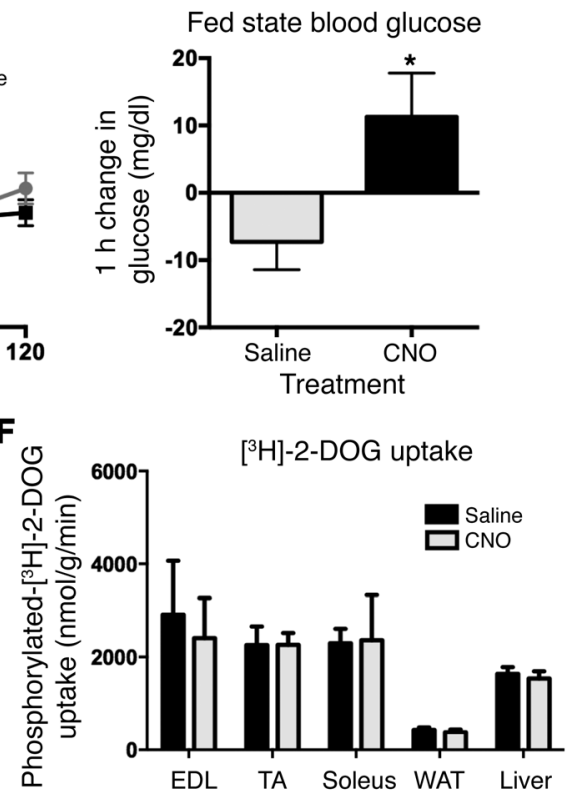

Figure 5. GCG neuronal activation produces a selective effect on glucose homeostasis. Insulin secretion and insulin sensitivity were not affected by CNO treatment (A, 2-way repeated measures ANOVA, $F_{1,11}=0.04572, P=0.8346 ; \mathbf{B}, 2$-way repeated measures ANOVA, $\left.F_{1,15}=0.4948, P=0.4926\right)$. Fed-state blood glucose levels showed a modest increase 1 hour after CNO delivery as compared with saline treatment (C, paired $t$ test, $\left.{ }^{*} P=0.038\right)$. i.p. pyruvate tolerance test showed a reduction in gluconeogenesis ( $\mathbf{D}, 2$-way repeated measures ANOVA, significant effect of treatment, $F_{5,35}=24.24,{ }^{*} P=0.0001 ; \mathbf{E}$, area under the curve, paired $t$ test, $\left.{ }^{*} P=0.0304\right)$. 2-Deoxyglucose uptake assay showed no effect of CNO on glucose disposal (F, $t$ test for each tissue sourced from saline- and CNO-treated animals, EDL $t=0.3492, P=0.7388$; TA $t=0.007964, P=0.9939$; soleus $t=0.893, P=0.398 ;$ WAT $t=0.5955, P=0.5732$; liver $t=0.4717, P=0.6538)$. Finally, fasted insulin levels were unchanged $(\mathbf{G}, t$ test, $n=8, t=0.056, P=0.956)$.

whereas RER and thus the ratio of carbohydrate and lipid fuel usage remained unchanged (Figure 6B; paired $t$ test, $t=0.1026, P=$ 0.9212). The decrease in energy expenditure was possibly related to reduced physical activity that was recorded after $\mathrm{CNO}$ injection (Figure 6C, paired $t$ tests $t=3.12$ and 3.18, $P=0.017$ and 0.016 , for $X$ and $Y$ ambulations, respectively). No effect of CNO treatment was observed on intrascapular brown adipose tissue (iBAT) activation, based on the fact that Ucp1, Pgcla, and Pparg gene expression was unchanged (Figure 6D; $U c p 1, t$ test, $t=0.94, P=0.398$; Pparg, $t$ test, $t=0.462, P=0.66$; Pgcla $t$ test, $t=0.07, P=0.94$ ). Based on the ability of GCG neuronal stimulation to produce a small acute reduction in food intake that does not extend beyond 4 hours of CNO injection (Figure 4D), we hypothesized that body weight would remain unchanged, unlike that observed following GLP-1R agonist treatment $(14,24)$. Indeed, after 14 days of HCD feeding followed by repeated injections of CNO (6 injections of $2 \mathrm{mg} / \mathrm{kg}$ CNO, every 8 hours), we failed to observe a change in body weight (Figure 6E; 2-way repeated measures ANOVA, $F_{1,6}=$ 1.336, $P=0.292$ ) and did not observe a change in food intake (data not shown). Prior work examining the activation of NPY peptideexpressing neurons in the arcuate (37), in contrast, has shown the ability of DREADD receptor activation to increase body weight following twice-daily injections of CNO over a 48-hour period, demonstrating that DREADD-driven stimulation can affect metabolic homeostasis in other experimental paradigms.

GCG neuronal activation is not sufficient for the development of a conditioned taste aversion. In addition to effects on feeding and metabolism, work has shown how central GLP-1R activation induces a conditioned taste aversion, independent of food intake regulation (20). To determine whether GCG neuronal stimulation can elicit taste aversion, we paired intraperitoneal $\mathrm{CNO}$ injection in GCG-Gq DREADD mice with the consumption of the novel tastant saccharin (0.15\% in water). CNO did not induce avoidance of the saccharin solution upon reintroduction in a 2-bottle choice test (Figure 6F; paired $t$ test, $t=0.7649, P=0.4733$ ), compared with controls. Injection of lithium chloride $(0.5 \mathrm{ml}$ of $0.15 \mathrm{M})$, however, subsequent to an exposure to a saccharin solution, produced aversion in a separate control group (Figure 6F). The amount of 
A

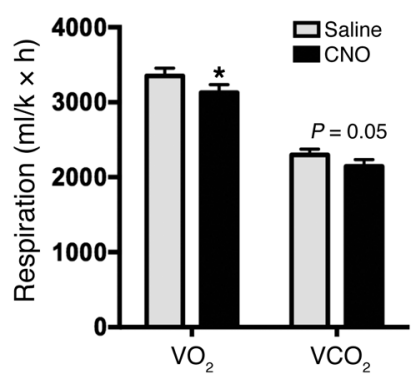

B

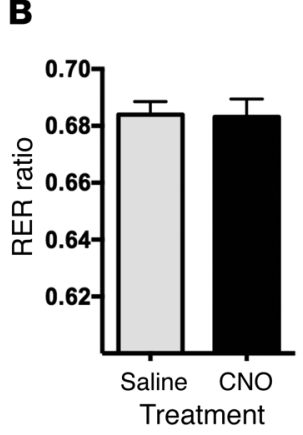

C

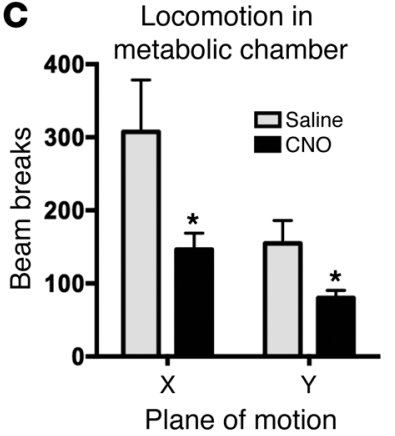

D

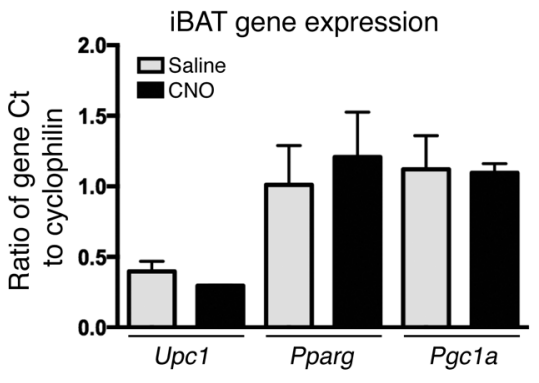

$\mathbf{E}$

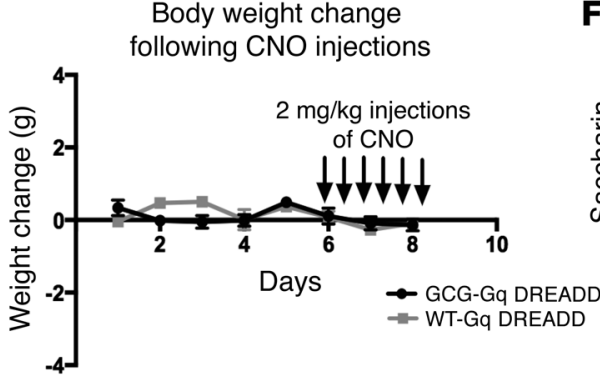

Body weight change following CNO injections

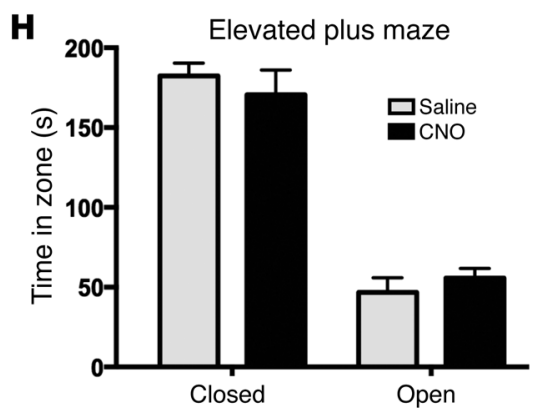

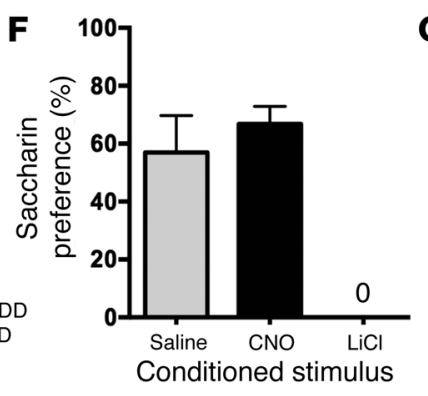

I

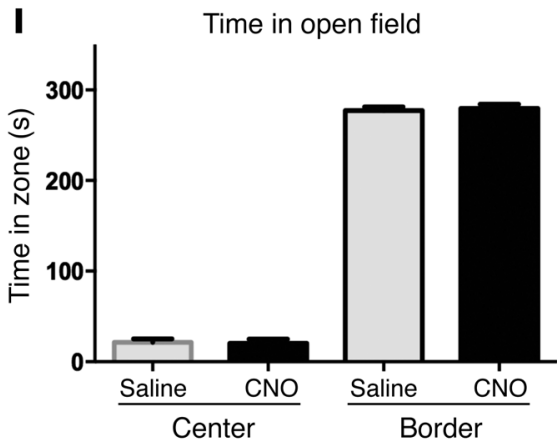

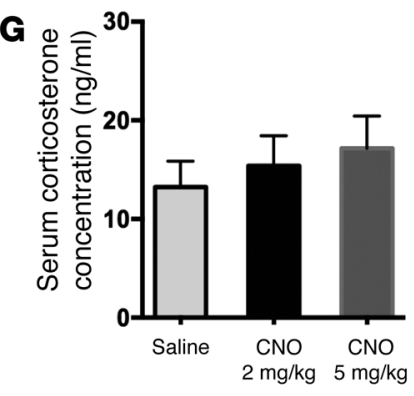

J

Figure 6. GCG neuronal activation alters metabolic rate and fasting locomotion, but has no effect on conditioned taste aversion, corticosterone levels, or anxiety-related behaviors. (A-C) GCG stimulation produces a small change in energy metabolism, with no effect on carbohydrate and fat utilization. CNO injection increased metabolic rate during the 3 hours after dark onset $\left(\mathbf{A}, \mathrm{VO}_{2}\right.$, paired $t$ test $n=8, t=2.661,{ }^{*} P=0.0324$; and VCO ${ }_{2}$ paired $t$ test $t=2.273, P=0.05$ ), with no change in RER (B, paired $t$ test, $t=0.1026, P=0.9212$ ). Activity was reduced (beam break counts in $\mathbf{C}$; paired $t$ tests $t=3.12$ and 3.18, ${ }^{*} P=0.017$ and 0.016 , for $X$ and $Y$ ambulations, respectively). (D) Ucp1, Pparg, and Pgc1a gene expression was unchanged in iBAT dissected from CNO- and saline-treated animals Ucp1 ( $t$ test $t=0.94, P=0.398$ ), Pparg ( $t$ test, $t=0.462, P=0.66$ ), Pgc1a ( $t$ test, $t=0.07, P=0.94)$. (E) Fourteen days of HCD feeding followed by CNO injections every 8 hours for 48 hours did not produce a change in body weight (2-way repeated measures ANOVA, $F_{1,6}=$ $1.336, P=0.292$ ). (F) CNO did not affect saccharin preference (paired $t$ test, $t=0.7649, P=0.4733$ ). Positive control LiCl produced a strong conditioned taste aversion (paired $t$ test, $t=5.816, P=0.0011$ ). (C) CNO did not increase serum corticosterone levels at 70 and 90 minutes, despite producing a reduction in food intake (shown in Figure 4A) (ANOVA, $F_{2,35}=0.1134, P=0.3834$ ). (H-J) CNO injection did not change performance in the elevated plus maze (H, $t$ test, open arms $t=0.7684, P=0.4534$; closed arms $t=0.6968, P=0.4954$ ), open field (I, time spent in open field, $t$ test, center $t=0.1924$, $P=0.8509$, border $t=0.3785, P=0.7123$; J, locomotor activity reflected in distance traveled in both border and central zones, $t$ test, border $t=0.5473$, $P=0.5991$, central $t=0.02015, P=0.9844)$.

saccharin solution consumed during the initial exposure prior to injections did not differ between the 3 groups.

GCG neuronal stimulation in lean animals has no effect on circulating corticosterone. Activation of GCG neurons produced a significant increase in PVN Fos staining, suggesting HPA axis activation. Unlike the effects on food intake, however, which may be due to action of GCG peptides in the arcuate, PVN, or other nuclei, CNO delivery had no effect on serum corticosterone levels in GCG-Gq DREADD mice measured at 9:00 am, between 60 and 80 minutes after CNO injection $(2 \mathrm{mg} / \mathrm{kg}: 15.4 \pm 3.0 \mathrm{ng} / \mathrm{ml}, 5 \mathrm{mg} / \mathrm{kg}: 17.15 \pm 3.3$ $\mathrm{ng} / \mathrm{ml})$, when compared with saline injection $(13.23 \pm 2.62 \mathrm{ng} / \mathrm{ml}$;
Figure 6G; ANOVA, $\left.F_{2,35}=0.1134, P=0.3834\right)$. The same CNO injections were able to significantly inhibit food intake when regular chow was returned to the animals in their home cages after tail vein blood draws (similar to that reported in Figure 4A).

GCG neuronal activation does not alter anxiety-like behaviors, as observed in the elevated plus maze and open field tests in lean mice. We tested the ability of GCG neuronal activation to modulate anxietylike behavior. Following either i.p. CNO or saline injections, GCG-Gq DREADD mice exhibited no differences in the elevated plus maze or the open field test. In the elevated plus maze test, the duration of time spent inside the open or closed arms was 
A

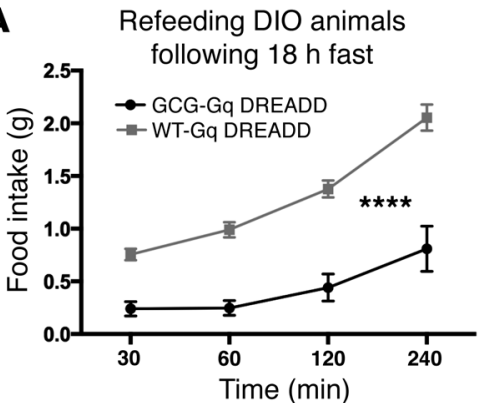

D Change in body composition following $2.0 \mathrm{mg} / \mathrm{kg} \mathrm{CNO}$

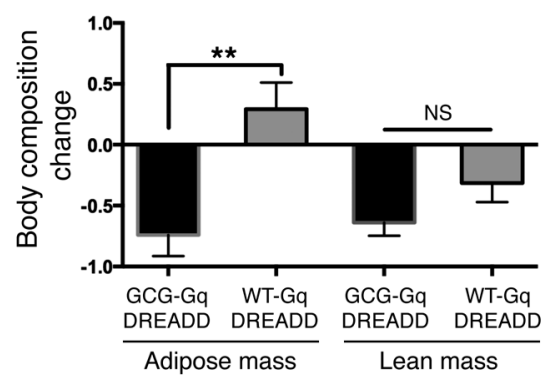

B

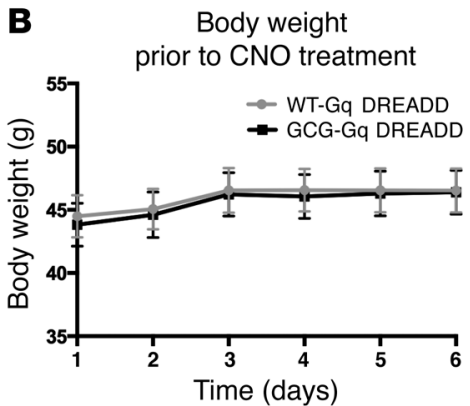

E

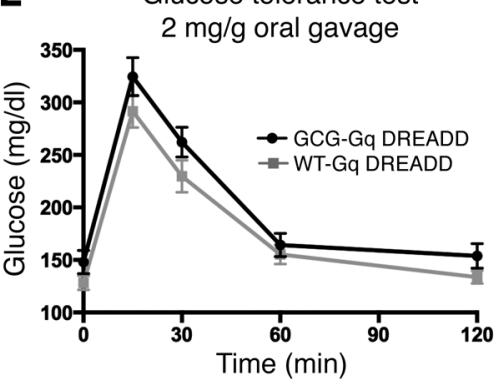

C Body weight change following $\mathrm{CNO}$ injections

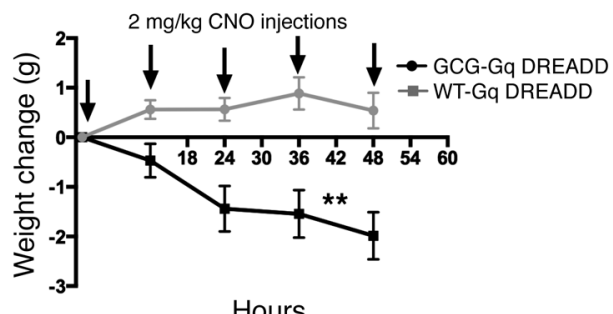

F

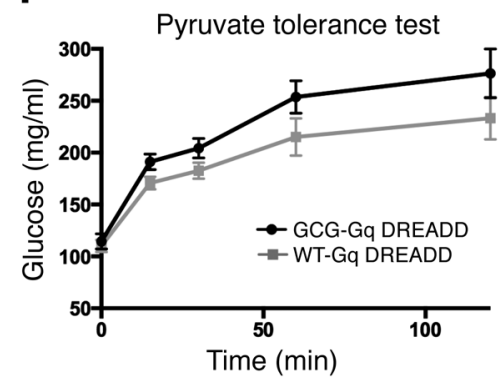

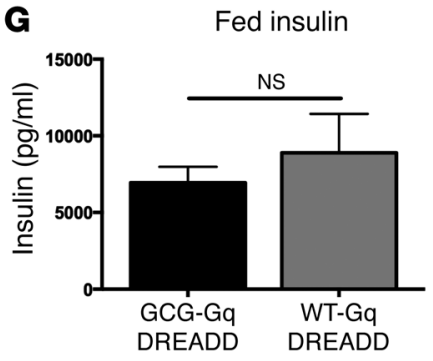

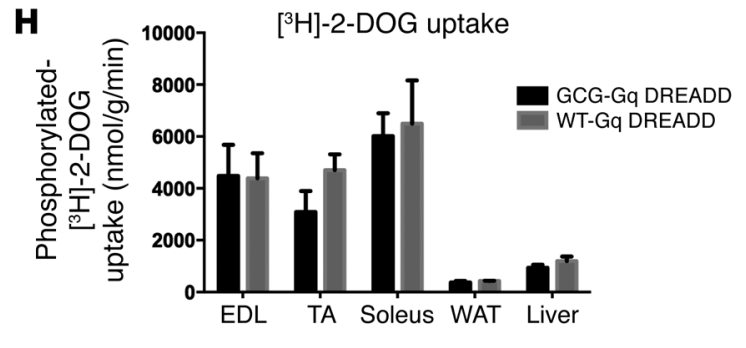

Figure 7. Anorexigenic effect of GCG neuronal stimulation is enhanced in DIO Gcg-Cre mice. $n=6-7$ control and GCG-Gq DREADD mice were fed HCD (Teklad TD88137) ad libitum for 5 months prior to testing. (A) CNO stimulation reduced food intake following an 18-hour fast compared with control CNO-injected littermates (2-way repeated measures ANOVA, significant effects of time $\left[F_{3,33}=72.91,{ }^{* * *} P<0.0001\right]$ and $C N O$ treatment $\left[F_{1,11}=36.04\right.$, $\left.{ }^{* * * *} P<0.0001\right]$ ). (B) Prior to testing the effects of CNO injection on body weight, control and GCG-Cq DREADD mice exhibited no difference in mass (2-way repeated measures ANOVA, $F_{1,11}=0.02455, P=0.8783$ ). (C) Regular injections of CNO spaced 12 hours apart significantly reduced body weight in the GCG-Gq DREADD animals but had no effect on controls (2-way repeated measures ANOVA, significant effects of time $\left[F_{4,44}=5.02,{ }^{* *} P=0.002\right]$ and CNO $\left[F_{1,11}=14.59,{ }^{* *} P=0.0028\right]$ ). (D) Fat mass was reduced ( $t$ test, $\left.t=3.76,{ }^{* *} P=0.0032\right)$, while no change in lean mass $(t$ test, $P=0.11)$ was seen. $(\mathbf{E}$ and $\mathbf{F})$ No effect of CNO injection on glucose homeostasis was observed. (E) Two-way repeated measures ANOVA, significant effect of time $\left(F_{444}=42.82, P<0.0001\right)$, no effect of CNO treatment $\left(F_{1,1}=4.182, P=0.655\right)$. (F) Two-way repeated measures ANOVA, significant effect of time $\left(F_{4,44}=86.73, P<0.0001\right)$, no effect of CNO treatment $\left(F_{1,11}=4.447, P=0.0587\right)$. No effect of CNO was observed on fed insulin levels ( $\mathbf{G}, t$ test $\left.t=0.758, P=0.464\right)$ or on glucose uptake (H, $n=3$ mice per group, $t$ test for each tissue sourced from CNO-treated WT and GCG-Gq DREADD animals, EDL $t=0.058, P=0.958 ;$ TA $t=1.604, P=0.1840$; soleus $t=0.2589, P=0.8085$; WAT $t=0.8964, P=0.4207$; liver $t=1.200, P=0.2964$ ).

not significantly different between saline and $\mathrm{CNO}$ treatments (Figure $6 \mathrm{H} ; t$ test, open arms $t=0.7684, P=0.4534$; closed arms $t=0.6968, P=0.4954)$. In the open field test, CNO and salinetreated groups showed no differences in time spent in the periphery or the center of the arena (Figure 6I; center $t=0.1924$, $P=0.8509$; border $t=0.3785, P=0.7123)$ and exhibited identical exploratory locomotor activity, reflected in distance traveled in both border and central zones (Figure 6J; border, $t$ test, $t=0.5473$, $P=0.5991$; central, $t$ test, $t=0.02015, P=0.9844$ ).

Development of obesity alters the ability of GCG neurons to drive changes in food intake, body weight, and glucose production. Finally, we investigated whether DIO alters the function of GCG neurons in the control of food intake and glucose homeostasis. Following
5 months of HCD feeding, we tested the ability of CNO to reduce food intake in obese mice, fasted for 18 hours then refed. Surprisingly, we found that $2 \mathrm{mg} / \mathrm{kg} \mathrm{CNO}$ produced an even larger reduction in food intake than observed in lean animals (Figure 7A; 2-way repeated measures ANOVA, significant effects of time $\left[F_{3,33}=\right.$ 72.91, $P<0.0001]$ and CNO treatment $\left.\left[F_{1,11}=36.04, P<0.0001\right]\right)$. Consequently, we tested the ability of repeated CNO injections to reduce body weight. Unlike in lean mice fed HCD, $2 \mathrm{mg} / \mathrm{kg} \mathrm{CNO}$ injections significantly reduced body weight in DIO mice over a 48-hour period (Figure 7C; 2-way repeated measures ANOVA, significant effects of time $\left[F_{4,44}=5.02, P=0.002\right]$ and $\mathrm{CNO}\left[F_{1,11}=\right.$ $14.59, P=0.0028])$, a reduction that was due to a loss of adiposity (Figure 7D, $t$ test, $P=0.0032$ ), with no significant change in lean 
mass (Figure 7D, $t$ test, $P=0.11$ ). Interestingly, when we tested the ability of CNO to reduce glucose production, we discovered that in DIO Gcg-Cre mice, CNO no longer reduced glucose production but showed a trend toward driving glucose production in the opposite direction (Figure 7F; 2-way repeated measures ANOVA, significant effect of time $\left[F_{4,44}=86.73, P<0.0001\right]$, no effect of $\mathrm{CNO}$ treatment $\left.\left[F_{1,11}=4.447, P=0.0587\right]\right)$. Furthermore, CNO-driven GCG neuronal activation had no effect on insulin sensitivity (Figure 7E; 2-way repeated measures ANOVA, significant effect of time $\left[F_{4,44}=42.82, P<0.0001\right]$, no effect of CNO treatment $\left[F_{1,11}=\right.$ $4.182, P=0.655]$ ) or on insulin production. Again, as was observed in lean mice, DREADD activation of GCG neurons did not affect fed serum insulin levels (Figure $7 \mathrm{G} ; t$ test, $t=0.758, P=0.464$ ) or glucose uptake into muscle, adipose, or liver tissue ( $t$ test; EDL $t=$ $0.058, P=0.958$; TA $t=1.604, P=0.1840$; soleus $t=0.2589, P=$ 0.8085 ; WAT $t=0.8964, P=0.4207$; liver $t=1.200, P=0.2964)$.

\section{Discussion}

Our data clarify the role of GCG neurons in the brain, as we demonstrate that this system is sufficient to produce changes in select metabolic parameters following stimulation. Specifically, we have shown how these neurons are sufficient in lean mice to modulate food intake while having no effect on corticosterone secretion, conditioned taste aversion, or exploratory behavior. Interestingly, while we also observed that GCG neuronal stimulation had minimal effects on glucose handling or insulin secretion, neuronal stimulation had potent effects on gluconeogenesis. Finally, our observed effect of GCG neuronal activation on metabolic rate occurred in the absence of any effects on brown fat activation or body weight. Interestingly, the effect of GCG neuronal stimulation was markedly different in DIO animals. While the ability of GCG neurons to reduce food intake and body weight was magnified, GCG neuronal control of gluconeogenesis was lost.

Our stimulation protocol involved the use of DREADD receptors to enhance the activity of GCG neurons, driving a small increase in neuronal activity. While we cannot determine whether we produced a change in GCG neuronal activity that exactly recapitulates the endogenous stimulation by afferent neurons, the degree of potentiation by CNO appears to match the ability of peptides that naturally activate GCG neurons, such as leptin and CCK, to produce a 2 -fold increase in neuronal action potential frequency and a small increase in resting membrane potential $(39,40)$. Thus, we believe that our work demonstrates the range of physiological effects that are possible following GCG neuronal activation by a physiologically relevant stimulus. It remains to be determined whether stronger stimulation (such as that which could be elicited by optogenetic activation) produces a more pronounced effect on physiology and whether GCG neurons receive such a stimulus in vivo.

GCG neuronal modulation of food intake. Our work shows for the first time to our knowledge that GCG neurons can independently suppress food intake when functionally activated by DREADD receptors, similar to the reported effects of GLP-1 or GLP-1 analog injections into either the cerebral ventricles or into the parenchyma of specific brain regions. Interestingly, the anorexigenic role of the GCG neurons does not appear to be dependent on the nutritional status of the animal, as has been reported previously (46). We had hypothesized that additional neurotransmitters released by GCG neurons may synergize with GLP-1 to reduce food intake following a fast. It is also possible that GCG neuronal stimulation may have negated the requirement for an elevation in leptin levels to produce anorexia in fasted animals (46), as we indeed saw that the suppression of food intake could be elicited in both satiated and fasted mice.

GCG neurons are ideally positioned to transduce satiety and energy influx signals to other brain regions controlling food intake such as the PVH and arcuate nucleus $(9,14,43,51)$. Indeed, we observed a modest increase in Fos IR in the PVH and the arcuate nucleus following $\mathrm{CNO}$ injection. In addition, other brain regions have been implicated in GLP-1-mediated modification of appetite and food intake, including the lateral parabrachial nucleus (16), lateral and dorsomedial hypothalamus (13), ventral tegmental area, and nucleus accumbens, all of which may be affected by the DREADD-driven activation of the hindbrain GCG neurons. These actions likely complement the ability of peripherally derived GLP-1 to produce satiety, acting at vagal afferents (6). Conditioned taste aversion, meanwhile, could not be recapitulated with DREADD-driven activation of GCG neurons $(18,19,24)$. Thus, activation of the central GCG neurons in the current study does not seem to lead to the development of nausea or malaise that would drive aversive conditioning to novel tastes. As prior work has shown that the sites that mediate satiety and taste aversion are distinct (20), it is possible that the endogenous activation of GCG neurons cannot produce malaise. Of course, it may be that stronger neuronal stimulation of the GCG neurons could produce taste aversion, a hypothesis that should be tested in future work. The potentiation of the anorexic effects of GCG neuronal stimulation in DIO mice when compared with lean animals (Figure 4D compared with Figure 7A) was surprising, given that ventromedial hypothalamic neurons, which form a principal target of the GCG cells, often become resistant to cues such as leptin and to neuronal stimulation in general that drive a reduction in food intake following HCD feeding (52). However, recent work has shown that peripherally administered liraglutide directly stimulates arcuate pro-opioid melanocortin (POMC) neurons while indirectly inhibiting neuropeptide Y/agouti-related peptide-expressing cells to reduce food intake and body weight in obese mice (14). Other work, meanwhile, has demonstrated that liraglutide can restore POMC neuron leptin sensitivity in obese animals (53), suggesting that POMC neurons could still play an important role, in addition to other GCG neuronal target sites, in the reduction of food intake and body weight in obese mice. Importantly, our work shows that GCG neurons may represent a therapeutic target for the treatment of obesity, as acute treatment reduced body weight significantly in DIO mice, over a 48-hour period of DREADD-driven Gcg neuronal activation. It remains to be determined whether GCG neurons are required in order to resist the development of obesity, a hypothesis we will test in future studies.

GCG neuronal control of glucose homeostasis. DREADD-driven activation of the hindbrain GCG neurons recapitulated only a subset of the glucoregulatory actions ascribed to central GLP-1Rs in lean animals. In the fed state, little effect of CNO-driven stimulation on blood glucose levels was seen. Insulin secretion and insulin sensitivity were unchanged following GCG neuronal activation. 
The main effects of GCG neuronal stimulation appear to be on gluconeogenesis, which was suppressed following neuronal activation and in the maintenance of glucose levels following a short-term fast. Interestingly, although no effects of GCG neuron activation on glucose clearance were observed, DREADD-driven activation of GCG neurons was sufficient to maintain normoglycemia in the context of short-term fasting for up to 3 hours. Our data suggest that, acutely, GCG neurons may in fact promote glucose production, possibly through an enhancement of sympathetic tone, as has been suggested previously (28). A significant portion of GCG neurons in the caudal medulla target sympathetic preganglionic neurons of the spinal cord (54). However, this postulated sympathetic activation would have to be tissue specific, as iBAT activation that might be expected to result from an increased sympathetic drive was not observed. The possibility that the centrally released neuropeptide GLP-1 may counteract the acute effect of the incretin gut hormone GLP-1 is intriguing and is with precedent, as the hindbrain has previously been shown to harbor neurons capable of driving glucose production in the periphery $(55,56)$.

The observation that GCG neuronal activation only modulated gluconeogenesis in a fasted lean animal was surprising to us, based on prior work showing an improvement in glucose uptake and glucose tolerance following GLP-1R and GLP-2R modulation (25-27). Specifically, the injection of GLP-1 into the arcuate nucleus but not the PVN reduces hepatic glucose production and glucose uptake $(25,26)$. Similar effects were also observed following GLP-2 injection ICV, with infusion increasing insulin sensitivity (increased peripheral glucose disposal) and reducing gluconeogenesis (27). This work is in agreement with other studies showing that central GLP-1R-dependent inhibition of hepatic glucose production occurs coincident with a suppression of skeletal muscle glucose uptake (48). Thus, published work demonstrates that GLP-1 action in the brain is sufficient to produce both insulin-dependent and insulin-independent actions on glucose homeostasis. In contrast, we believe our data suggest that DREADD-driven GCG neuronal activation is therefore only sufficient to modulate a subset of the possible actions of these cells on glucose homeostasis. Our data suggest that the insulin-sensitizing actions of GCG peptides and gluconeogenic actions of these peptides can be functionally uncoupled, which could be accomplished by different populations of neurons mediating changes in glucose uptake, insulin release, and gluconeogenesis in the context of GLP-1R agonist administration. Alternately, a single population of neurons innervated by GCG neurons may drive glucose handling and production, with actions dependent upon the degree of GCG neuronal activation.

Unlike in lean animals, in DIO mice, there was no effect of GCG neuronal stimulation on gluconeogenesis. As prior work suggests that arcuate neurons mediate the effects of GLP-1 on glucose homeostasis (26), the resistance to the actions of GCG neurons in driving a reduction in glucose production likely occurs in downstream hypothalamic neurons that regulate peripheral hepatic glucose production $(52,57)$. This effect was surprising, however, given data cited earlier that GLP-1R agonism can reverse obesity-driven neuronal insensitivity to hormones such as leptin in arcuate POMC neurons $(14,53)$ to produce a change in food intake. Thus, our data (and those of others; ref. 26) suggest that the GCG neuronal regulation of food intake and glucose homeostasis is mediated by sep- arate populations of neurons that show differential sensitivity to GCG neuronal stimulation in the setting of obesity.

GCG neuronal control of metabolic rate. In addition to effects on glucose homeostasis and food intake, GCG peptide signaling has been hypothesized to produce a change in brown fat activation and respiration rate. Interestingly, both an increase and decrease in metabolic rate have been reported, making it difficult to predict the actions of the endogenous GCG neuronal system. Chronic GLP-1 infusion into the CNS has been shown to lead to transcriptional activation of $\mathrm{BBAT}$ and glucose/triglyceride uptake $(49,58)$ resulting from an increase in sympathetic outflow driven by GLP-1R signaling. Other data, however, have shown that the GLP-1R agonist EX-4 can also produce a lowering of $\mathrm{VO}_{2}$. Similarly, a reduction in GLP-1R signaling has been shown to increase metabolic rate, in both the GLP-1R-knockout model and following infusion of the GLP-1R antagonist EX-9 into the brain (47). In several prior studies, locomotion was also affected by GLP-1R modulation. We believe that our data clarify the role of the endogenous neuronal GCG peptide releasing system following acute activation. Clearly, GCG neurons act to reduce metabolic rate while also reducing locomotion in a context-dependent manner. How GCG neurons produce this effect is less well understood when compared with the glucoregulatory and food intake actions of the GCG peptides. As with actions of the GCG neurons on food intake, multiple sites could be mediating the effects of the GCG neurons on locomotor activity, such as the POMC arcuate neurons and neurons of the nucleus accumbens $(14,21,59)$.

It was surprising that while ambulatory activity was altered in mice in the Oxymax chambers during the subjective dark phase, no effect was observed in the open field test conducted during the light phase. Taking both of these results into consideration, we believe that it is possible that the change in activity while in the metabolic chambers is related to either a reduced foraging drive that underlies the reduced food intake with GCG neuronal stimulation or to an alteration in nocturnal activity. Further studies will be required to investigate this effect.

While a change in locomotion may contribute to the change in metabolic rate, it is unlikely that effects on metabolic rate were influenced by any modulation of iBAT activity. Again, similar to the effects on glucose homeostasis, lack of an effect of GCG neuronal stimulation on iBAT activity was unexpected, as other work has shown the ability of acute CNS infusion of GLP-1 to increase iBAT activation $(49,58)$.

GCG neuronal stimulation and body weight regulation. Based on our data showing both a reduction in food intake (that did not extend beyond 3 hours after CNO injection; data not shown) and a reduction in energy expenditure, we were not surprised that CNO stimulation of GCG neurons did not produce a change in body weight in lean animals. Interestingly, GCG neuronal activation produced a significant effect on body weight in DIO mice, lowering adipose levels following DREADD activation, as has been reported for the actions of liraglutide on obese mice $(14,49)$ at arcuate POMC and NPY/AgRP neurons $(14,26)$. Our work therefore clarifies the ability of endogenous GCG neuronal stimulation to recapitulate the effects of peripherally administered liraglutide on body weight selectively in obese animals, producing weight loss of a similar magnitude to that observed in obese humans (60). 
GCG neuronal modulation of HPA axis activation and anxiety-like behavior. Finally, in addition to effects on food intake and glucose homeostasis, we expected that, based on its excitatory input to the PVH, GCG neuronal stimulation would lead to a robust secretion of corticosterone. In rats, GLP-1 infusion into the third ventricle or the PVN lead to a robust ACTH and corticosterone response (34). As a significant proportion of corticotropin-releasing hormone-containing (CRH-containing) parvocellular neurons are innervated by GCG neurons $(9,10,61)$, we expected to observe a rise in serum corticosterone levels after $\mathrm{CNO}$ injection. Our observations that food intake following tail blood draws was reduced in CNO-treated animals and that $\mathrm{CNO}$ induced a modest increase in Fos expression in the PVN would also suggest that our stimulation paradigm would ultimately result in increased corticosterone secretion. This, however, did not occur and could have resulted from an insufficient degree of GCG neuron activation to elicit a net strong excitatory effect on the $\mathrm{CRH}$ neurons of the PVH. Last, despite the reported role of the central GCG system in the stress response to novel and open spaces $(34,62)$, we did not see effects of GCG stimulation on open field or elevated plus maze performance. The underlying reasons for this discrepancy could be species differences in an anxiety- or open space aversion-promoting role for GCG neurons, which was demonstrated in rats to involve the central nucleus of the amygdala (34). Further testing is needed to reveal whether hindbrain GCG neurons provide significant contributions to neuroendocrine and behavioral stress responses to either aversive interoceptive and exteroceptive stimuli.

In summary, our DREADD-driven moderate stimulation paradigm reveals the ability of GCG neurons to drive a subset of the physiological effects ascribed to GLP-1R agonists in the brain. Even though neuronal activation engages hypothalamic target nuclei and reduces food intake, this mode of activation was not sufficient to enhance corticosterone secretion, modulate anxiety behavior, reduce glucose uptake, affect insulin sensitivity, or change the development of a conditioned taste aversion. Thus, it is possible that a moderate level of GCG neuron activation may produce an inhibition or termination of feeding and reduction in glucose production while avoiding other effects ascribed to central agonism of receptors that bind GCG-derived peptides. In the setting of DIO, GCG neuronal stimulation no longer affects gluconeogenesis but significantly reduces food intake, leading to a reduction in body weight and adiposity.

\section{Methods}

Animals. Mice were housed on a 6:00 am/off 6:00 pm/on light cycle. Mice were fed ad libitum Harlan Chow (catalog 7912), $3.1 \mathrm{kcal} / \mathrm{g}, 5.8 \%$ of kcal from fat and water, unless stated otherwise.

Generation of the Gcg-Cre mouse line. Cre recombinase was inserted into the Gcg gene locus of BAC RP23-242F22 at the ATG start codon using previously described recombineering methods (63) (Figure 1). The BAC transgene was then injected into pronuclei from C57BL/6J animals (Jackson Laboratory, strain 000664).

Mice were genotyped using primers $\mathrm{Cr}^{+} 5^{\prime}$-GTGAAACAGCATTGCTGTCAC-3', Cre- 5'-TGCTTCTGTCCGTTTGCCGGT- $3^{\prime}$ and the control primers run in the same reaction, M176 5'-GGTCAGCCTAATTAGCTCTGTCAT and M177 5'-GATCTCCAGCTCCTCCTCTGTCT- $3^{\prime}\left(\mathrm{Tm}=60^{\circ} \mathrm{C}\right)$. tdTomato reporter mice were genotyped with primers WT forward 5'-AAGGGAGCTGCAGTGGAGTA-3', WT reverse 5'-CCGAAAATCTGTGGGAAGTC-3', mutant reverse 5'-GGCATTAAAGCAGCGTATCC-3', mutant forward 5'-CTGTTCCTGTACGGCATGG-3'.

Quantitative PCR. Quantitative PCR was performed as described previously (64), using the following primer pairs to detect the control gene cyclophilin and the iBAT-expressed transcripts cyclophilin': CGATGACGAGCCCTTGG and cyclophilin: TCTGCTGTCTTTGGAACTTTGTC, Pparg : GATGCAAGGGTTTTTTCCGAAG and Pparg: CAAGGCACTTCTGAAACCGACA, $P g c 1 a^{+}$: CCCTGCCATTGTTAAGAC and Pgcla: TGCTGCTGTTCCTGTTTT, Ucp1 $1^{+}$: ACTGCCACACCTCCAGTCATT and Ucp1 ${ }^{-}$: CTTTGCCTCACTCAGGATTGG.

Surgery. Six- to 8-week-old Gcg-Cre mice were injected with AAV2/5-hSyn-DIO-hM ${ }_{3}$ Dq (Gq)-mCherry (encoding Gq DREADD) (University of North Carolina Gene Therapy Core) using pressure injection into the NTS (200 nl virus/side). Mice received intramuscular ketamine $(60 \mathrm{mg} / \mathrm{kg})$ and xylazine $(10 \mathrm{mg} / \mathrm{kg})$ and upon full anesthesia were immobilized in a stereotaxic frame (David Kopf Instruments). The dorsal neck musculature was exposed and dissected via midline incision to expose the dorsal surface of the caudal brainstem and the floor of the fourth ventricle. The dura mater was punctured with a $30 \mathrm{G}$ needle tip at $0.5 \mathrm{~mm}$ both left and right to the caudal limit of the area postrema, and a glass micropipette (tip diameter 20-30 $\mu \mathrm{m})$ filled with the AAV vector was lowered $0.4 \mathrm{~mm}$ ventral to the dorsal surface of the brain. A total of $200 \mathrm{nl} \mathrm{AAV}$ vector solution was delivered by pressure injection (MPPI-2, Applied Scientific Instrumentation) over a period of 5 minutes, after which the micropipette tip was left in situ for 2 minutes and then slowly removed. The incision was then closed with sutures, and the animals received ketoprofen (5 $\mathrm{mg} / \mathrm{kg}$, s.c.) during recovery from anesthesia. The Cre-negative (WT) littermates received sham surgery. Mice were singly housed for 3-5 days after surgery before being re-housed with cage mates.

Feeding and body weight assays. To assess daytime and nighttime food intake, food (Harlan, catalog 7912, $3.1 \mathrm{kcal} / \mathrm{g}, 5.8 \%$ of kcal from fat) was removed 2 hours prior to injection of $\mathrm{CNO}(2 \mathrm{mg} / \mathrm{kg})$ or saline, at either 11:00 am or 5:00 pm. Food consumption was determined 2 or 3 hours later. To assess fasting-driven food intake, food was removed at 4:00 pm, with mice receiving $\mathrm{CNO}$ or saline injections the next morning between 9:00 and 9:30 am. Thirty minutes later, food intake was measured at $30,60,120$, and 240 minutes. To assess suppression of palatable HCD intake, animals were first exposed to the diet (Research Diets Inc., catalog D12331, $5.56 \mathrm{kcal} / \mathrm{g}, 58 \%$ of $\mathrm{kcal}$ from fat) to prevent neophobia. The next day, mice received either $2 \mathrm{mg} / \mathrm{kg}$ CNO or saline injections 2 hours after lights on and were then tested at 15 and 30 minutes. Each experimental procedure was repeated with $\mathrm{CNO}$ and saline injections reversed, after a 3-day recovery.

Acute regulation of body weight following acute $H C D$. Body weight was determined daily in mice fed ad libitum on HCD for 2 weeks. Following the 14 days of HCD feeding, animals were then injected either with saline in the control group or with $2 \mathrm{mg} / \mathrm{kg}$ CNO every 8 hours for 2 days.

Induction of DIO. Following surgery, mice were fed ad libitum on HCD (Teklad TD.88137) for 5 months prior to testing for changes in food intake, body weight, and glucose homeostasis.

Measurement of blood glucose and insulin levels; and glucose, pyruvate, and insulin tolerance assays. Mice were handled for 3 days prior to testing. Tail blood was measured using a handheld blood glucose monitor (NovaMax). For the glucose and pyruvate tolerance tests, mice 
were fasted for 18 hours. Animals then received i.p. injections of saline or CNO $(2 \mathrm{mg} / \mathrm{kg}) 30$ minutes prior to sampling. Mice were gavaged with dextrose ( $2 \mathrm{mg} / \mathrm{g}$ body weight, $25 \%$ in water for glucose tolerance testing) or were i.p. injected with pyruvate ( $2 \mathrm{mg} / \mathrm{g}$ body weight, $25 \%$ in water), with glucose analyzed $0,15,30,60$, and 120 minutes later. Treatments were switched and mice retested 3 days later.

Acute effects of GCG stimulation on glucose levels. Food was removed 2 hours prior to testing. Tail blood was measured immediately prior to injection of $\mathrm{CNO}$ or saline and then 1 hour thereafter. Three days later, treatments were switched, and the assay was repeated.

Insulin tolerance test. Food was removed 2 hours prior to $\mathrm{CNO} /$ saline injection. Thirty minutes after CNO or saline injection, Humulin-R (Eli Lilly and Co.) was injected at $0.75 \mathrm{U} / \mathrm{kg}$ body weight in a volume of $4 \mathrm{l} / \mathrm{g}$ i.p. Tail blood glucose was tested at $0,15,30,60$, and 120 minutes after insulin treatment. Three days later, treatments were switched, and the assay was repeated. Insulin was measured using a mouse insulin ELISA (Crystal Chem) as per the manufacturer's instructions.

$\left[{ }^{3} \mathrm{H}\right]-2-D O G$ uptake assay: in vivo glucose transport assessment during glucose tolerance testing. The 2-deoxyglucose (2-DOG) uptake assay was conducted as described previously $(65,66)$. Briefly, 2-deoxy-D-[1,2${ }^{3} \mathrm{H}$ ]-glucose (Amersham) was mixed with $20 \%$ dextrose to deliver $2 \mathrm{~g} /$ $\mathrm{kg}$ glucose and $10 \mu \mathrm{Ci}$ i.p. to each mouse. Thirty minutes prior to testing, mice were injected with either CNO or saline. Tail blood was sampled and glucose measured at times $0,15,30,45,60$, and 120 minutes. Glucose-specific activity from plasma was determined by scintillation counting. Area under the curve for the glucose-specific activity was determined following blood draws and used in calculations described below. To determine the uptake of 2-DOG-6-phosphate, at $120 \mathrm{~min}$ utes, animals were euthanized and tissues extracted and homogenized in $2 \mathrm{ml}$ water. $1.6 \mathrm{ml}$ was mixed with $7 \%$ perchloric acid. Following $\mathrm{KHCO}_{3}$ treatment, protein levels were determined by Bradford assay. A fraction of the supernatant was used to determine total ${ }^{3} \mathrm{H}$ radioactivity by passing through an ion exchange column to extract labeled 2-DOG-6-phosphate. Radioactivity was then eluted and counted using a scintillation counter. This counted fraction was compared with another fraction of the supernatant to obtain unfiltered ${ }^{3} \mathrm{H}$ counts. The difference between the two samples was used to calculate accumulated 2-deoxy-D-[1,2-3 H]-glucose-6-phosphate. Finally, 2-DOG uptake was determined by dividing the determined accumulated 2-deoxy-D-[1,2$\left.{ }^{3} \mathrm{H}\right]$-glucose-6-phosphate by the plasma glucose-specific activity area under the curve and by the protein concentration.

Electrophysiology. Coronal brain slices $(300 \mu \mathrm{m})$ containing the NTS were prepared from AAV hM3Dq-DREADD-injected mice $(n=4)$. Recordings were performed as described previously (67).

Indirect calorimetry and ambulatory activity. Eight mice were transferred into metabolic cages (Oxymax Lab Animal Monitoring System, Columbus Instruments) and housed for 5 days. After 3 days of acclimation, food was removed 2 hours prior to dark onset, and CNO and saline injections (4 mice each) were given 1 hour prior to dark onset. $\mathrm{VO}_{2}, \mathrm{VCO}_{2}, \mathrm{RER}$, and ambulatory activity (via infrared photocell beam interruption) were measured over a 3-hour period after dark onset, after which food was returned. The next day, the groups of mice undergoing either $\mathrm{CNO}$ and saline treatment were switched.

Conditioned taste aversion. The assay was performed as described previously (24), with mice acclimated to drinking tubes 2 days prior to testing. GCG-Gq DREADD mice were deprived of water for 18-20 hours and then given access to $0.15 \%$ saccharin solution for 2 hours.
The solution was presented in drinking tubes made from 10-ml pipettes. They then received injection of either saline or CNO (2 mg/ $\mathrm{kg}$ i.p.). A third group of WT siblings received lithium chloride ( $\mathrm{LiCl}$, $0.15 \mathrm{M}$ in $0.5 \mathrm{ml}$ saline s.c.) to serve as a positive control. The saccharin drinking tubes were then removed and replaced with tubes with water that were placed on the cage side opposite that of the saccharin-filled tubes. The following day the animals were again water deprived overnight, and approximately 20 hours later water and saccharin-filled drinking tubes were returned to their previous locations. Fluid intake was measured 2 hours later.

Corticosterone assay. Food was removed 2 hours prior to i.p. injection with CNO or saline. One hour after injection, mice were restrained, and tail vein blood collected. Serum was analyzed for corticosterone content using a solid-phase ELISA according to the manufacturer's protocol (IBL-America).

Anxiety-related behaviors: elevated plus maze and open field. Experiments were performed as described previously (68). Mice were habituated to the behavioral testing room for 3 days prior to the procedures. Each mouse was injected with either saline or $2 \mathrm{mg} / \mathrm{kg}$ CNO i.p. 30 minutes prior to the testing, while food was removed 2 hours prior.

Brain tissue preparation and immunohistochemistry. Immunohistochemistry was performed as previously described (69). CNO (2 mg/ $\mathrm{kg}, n=12$ ) or saline ( $n=2)$ was injected 2 hours prior to sacrifice by transcardial perfusion fixation. A group of WT siblings was also included to serve as controls. After anesthesia, mice were briefly flushed with buffered saline, followed by perfusion with $4 \%$ paraformaldehyde in $0.1 \mathrm{M}$ phosphate buffer and 15\% (v/v) of saturated picric acid. Brains were dissected and sectioned at $40 \mu \mathrm{m}$ on a Vibratome. Immunoperoxidase staining was used to visualize (i) Fos and GLP-1, (ii) Fos and DsRed, and (iii) GLP-1 and DsRed coexpression. All primary and secondary antibody solutions were made in $0.01 \mathrm{M}$ PBS containing $0.5 \%$ Triton $\mathrm{X}-100,1 \%$ normal goat serum, and $0.1 \%$ sodium azide with the following dilutions: rabbit anti-Fos: 50,000 $\times$ (Ab-5, Oncogene/Millipore, catalog PC38), rabbit anti-GLP-1: 5,000× (Bachem/Peninsula Laboratories Inc., catalog T-4363), and rabbit anti-DsRed: 5,000× (Living Colors Clontech, catalog 632496). After 48 hours incubation in primary antibody, sections were washed in PBS and transferred into biotinylated secondary antibody (goat anti-rabbit IgG at 1,000× dilution, Jackson ImmunoResearch Laboratories Inc.) followed by ABC Elite kit (Vector Laboratories). For every combination, the first marker was visualized with nickel-enhanced DAB to yield a black color, while the second marker was visualized with regular DAB, yielding a reddish-brown color.

To evaluate the impact on hypothalamic targets implicated in the control of feeding and energy balance, forebrains of the GCG-Gq DREADD mice were sectioned and processed for Fos and GLP-1 IR as described above. Fos IR appeared as black-stained cellular nuclei, whereas GLP-1 IR emerged as brown-colored axons and terminal boutons.

To determine the specificity of fluorescent reporter protein expression in GCG neurons, $\mathrm{Gcg}$-Cre tdTomato mice were sacrificed by perfusion fixation, brains were sectioned, and the hindbrain sections were processed for GLP-1 immunofluorescence as above using Alexa Fluor 488-conjugated goat anti-rabbit (Molecular Probes) as the secondary antibody to enable dual fluorescence detection of tdTomato and GLP-1. The remainder of the brain was sectioned and processed to yield DsRed immunoperoxidase staining (as described above) using nickel-enhanced DAB as a chromogen. 
Statistics. All data were subjected to statistical analysis in GraphPad Prism 6. When WT and transgene GCG-Gq DREADD mice were compared, 2-tailed unpaired $t$ tests were performed. Time course tests were analyzed with 2-way repeated measures ANOVA with treatment and time points as independent variables. When the same mice received alternating $\mathrm{CNO}$ and saline treatments, paired 2-tailed $t$ tests were used to evaluate data for significance. Data from once-performed behavioral tests, such as the open field and elevated plus maze, were analyzed with unpaired 2-tailed $t$ tests. Data are expressed as mean \pm SEM. Statistical significance was considered with $P<0.05$.

Study approval. All animal procedures were approved by the Institutional Animal Care and Use Committee of the University of Virginia and conducted in accordance with its guidelines.

\section{Author contributions}

RPG conducted experiments, analyzed data, and wrote the manuscript. BAN conducted experiments and analyzed data. MO conducted experiments, analyzed data, and wrote the manuscript. VR conducted experiments and analyzed data. DMW conducted experiments and analyzed data. PSL analyzed data and conducted experiments. MN conducted experiments and analyzed data. TY conducted experiments and analyzed data. YH conducted experiments and analyzed data. IGS conducted experiments and analyzed data. TEH conducted experiments and analyzed data. MKP conducted experiments, analyzed data, and wrote the manuscript. KWW coordinated experiments and wrote the manuscript. MMS planned the experiments, conducted the experiments, analyzed the data, and wrote the manuscript.

\section{Acknowledgments}

The authors acknowledge grants from the National Institute on Drug Abuse, NIH (R00 DA024719) and the Virginia Fund for Healthy Youth (both to MMS); the National Institute of Diabetes and Digestive and Kidney Diseases, NIH (R01 DK100699 to KWW, F32 DK102294 to DMW, and R01 DK101946 to TEH); and the China Scholarship Council (to TY).

Address correspondence to: Michael M. Scott, Department of Pharmacology, School of Medicine, University of Virginia, 1340 Jefferson Park Avenue, Jordan Hall, Room 5050, Charlottesville, Virginia 22908, USA. Phone: 434.243.1920; E-mail: michael. scott@virginia.edu.
1. Sandoval DA, D'Alessio DA. Physiology of proglucagon peptides: role of glucagon and GLP-1 in health and disease. Physiol Rev. 2015;95(2):513-548.

2. Kreymann B, Williams G, Ghatei MA, Bloom SR. Glucagon-like peptide-17-36: a physiological incretin in man. Lancet. 1987;2(8571):1300-1304.

3. Woerle HJ, Carneiro L, Derani A, Göke B, Schirra $\mathrm{J}$. The role of endogenous incretin secretion as amplifier of glucose-stimulated insulin secretion in healthy subjects and patients with type 2 diabetes. Diabetes. 2012;61(9):2349-2358.

4. Kieffer TJ, Habener JF. The glucagon-like peptides. Endocr Rev. 1999;20(6):876-913.

5. Ritzel R, Orskov C, Holst JJ, Nauck MA. Pharmacokinetic, insulinotropic, and glucagonostatic properties of GLP-1 [7-36 amide] after subcutaneous injection in healthy volunteers. Dose-response-relationships. Diabetologia. 1995;38(6):720-725.

6. Krieger JP, Arnold M, Pettersen KG, Lossel P, Langhans W, Lee SJ. Knockdown of GLP-1 receptors in vagal afferents affects normal food intake and glycemia. Diabetes. 2016;65(1):34-43.

7. Turton MD, et al. A role for glucagon-like peptide- 1 in the central regulation of feeding. Nature. 1996;379(6560):69-72.

8. Merchenthaler I, Lane M, Shughrue P. Distribution of pre-pro-glucagon and glucagon-like peptide-1 receptor messenger RNAs in the rat central nervous system. JComp Neurol. 1999;403(2):261-280.

9. Llewellyn-Smith IJ, Reimann F, Gribble FM, Trapp S. Preproglucagon neurons project widely to autonomic control areas in the mouse brain. Neuroscience. 2011;180:111-121.

10. Richards $\mathrm{P}$, et al. Identification and characterization of GLP-1 receptor-expressing cells using a new transgenic mouse model. Diabetes. 2014;63(4):1224-1233.

11. Zheng H, Stornetta RL, Agassandian K, Rinaman L. Glutamatergic phenotype of glucagon-like peptide 1 neurons in the caudal nucleus of the solitary tract in rats. Brain Struct Funct. 2015;220(5):3011-3022.

12. Zheng H, Cai L, Rinaman L. Distribution of glucagon-like peptide 1-immunopositive neurons in human caudal medulla. Brain Struct Funct. 2015;220(2):1213-1219.

13. Schick RR, Zimmermann JP, vorm Walde T, Schusdziarra V. Peptides that regulate food intake: glucagon-like peptide 1-(7-36) amide acts at lateral and medial hypothalamic sites to suppress feeding in rats. Am J Physiol Regul Integr Comp Physiol. 2003;284(6):R1427-R1435.

14. Secher A, et al. The arcuate nucleus mediates GLP-1 receptor agonist liraglutide-dependent weight loss. JClin Invest. 2014;124(10):4473-4488.

15. Dossat AM, Diaz R, Gallo L, Panagos A, Kay K, Williams DL. Nucleus accumbens GLP-1 receptors influence meal size and palatability. Am JPhysiol Endocrinol Metab. 2013;304(12):E1314-E1320.

16. Alhadeff AL, Baird JP, Swick JC, Hayes MR, Grill HJ. Glucagon-like peptide-1 receptor signaling in the lateral parabrachial nucleus contributes to the control of food intake and motivation to feed. Neuropsychopharmacology. 2014;39(9):2233-2243.

17. Alhadeff AL, Grill HJ. Hindbrain nucleus tractus solitarius glucagon-like peptide-1 receptor signaling reduces appetitive and motivational aspects of feeding. Am J Physiol Regul Integr Comp Physiol. 2014;307(4):R465-R470.

18. Kanoski SE, Rupprecht LE, Fortin SM, De Jonghe BC, Hayes MR. The role of nausea in food intake and body weight suppression by peripheral GLP-1 receptor agonists, exendin-4 and liraglutide. $\mathrm{Neu}$ ropharmacology. 2012;62(5-6):1916-1927.

19. Lachey JL, D'Alessio DA, Rinaman L, Elmquist JK, Drucker DJ, Seeley RJ. The role of central glucagon-like peptide- 1 in mediating the effects of visceral illness: differential effects in rats and mice. Endocrinology. 2005;146(1):458-462.

20. Kinzig KP, D’Alessio DA, Seeley RJ. The diverse roles of specific GLP-1 receptors in the control of food intake and the response to visceral illness. JNeurosci. 2002;22(23):10470-10476.

21. Dossat AM, Lilly N, Kay K, Williams DL. Glucagon-like peptide 1 receptors in nucleus accumbens affect food intake. J Neurosci. 2011;31(41):14453-14457.

22. Richard JE, Anderberg RH, Göteson A, Gribble FM, Reimann F, Skibicka KP. Activation of the GLP-1 receptors in the nucleus of the solitary tract reduces food reward behavior and targets the mesolimbic system. PLoS One. 2015;10(3):e0119034.

23. Swick JC, et al. Parabrachial nucleus contributions to glucagon-like peptide-1 receptor agonist-induced hypophagia. Neuropsychopharmacology. 2015;40(8):2001-2014.

24. Sisley S, Gutierrez-Aguilar R, Scott M, D'Alessio DA, Sandoval DA, Seeley RJ. Neuronal GLP1R mediates liraglutide's anorectic but not glucose-lowering effect. JClin Invest. 2014;124(6):2456-2463.

25. Sandoval D. CNS GLP-1 regulation of peripheral glucose homeostasis. Physiol Behav. 2008;94(5):670-674.

26. Sandoval DA, Bagnol D, Woods SC, D'Alessio DA, Seeley RJ. Arcuate glucagon-like peptide 1 receptors regulate glucose homeostasis but not food intake. Diabetes. 2008;57(8):2046-2054.

27. Shi X, et al. Central GLP-2 enhances hepatic insulin sensitivity via activating PI3K signaling in POMC neurons. Cell Metab. 2013;18(1):86-98.

28. Pérez-Tilve D, et al. Exendin-4 increases blood glucose levels acutely in rats by activation of the sympathetic nervous system. Am J Physiol Endocrinol Metab. 2010;298(5):E1088-E1096.

29. Yamamoto H, et al. Glucagon-like peptide-1 receptor stimulation increases blood pressure and heart rate and activates autonomic regulatory neurons. J Clin Invest. 2002;110(1):43-52.

30. Rinaman L. Interoceptive stress activates glucagon-like peptide-1 neurons that project to 
the hypothalamus. Am J Physiol. 1999; 277(2 Pt 2):R582-R590.

31. Rinaman L. A functional role for central glucagonlike peptide-1 receptors in lithium chloride-induced anorexia. Am JPhysiol. 1999; 277(5 Pt 2):R1537-R1540.

32. Tauchi M, Zhang R, D'Alessio DA, Seeley RJ, Herman JP. Role of central glucagon-like peptide-1 in hypothalamo-pituitary-adrenocortical facilitation following chronic stress. Exp Neurol. 2008;210(2):458-466.

33. Gil-Lozano M, et al. GLP-1(7-36)-amide and Exendin- 4 stimulate the HPA axis in rodents and humans. Endocrinology. 2010;151(6):2629-2640.

34. Kinzig KP, et al. CNS glucagon-like peptide-1 receptors mediate endocrine and anxiety responses to interoceptive and psychogenic stressors. J Neurosci. 2003;23(15):6163-6170.

35. Maniscalco JW, Zheng H, Gordon PJ, Rinaman L. Negative energy balance blocks neural and behavioral responses to acute stress by "silencing" central glucagon-like peptide 1 signaling in rats. J Neurosci. 2015;35(30):10701-10714.

36. Heppner KM, Perez-Tilve D. GLP-1 based therapeutics: simultaneously combating T2DM and obesity. Front Neurosci. 2015;9:92.

37. Krashes MJ, et al. Rapid, reversible activation of AgRP neurons drives feeding behavior in mice. J Clin Invest. 2011;121(4):1424-1428.

38. Alexander GM, et al. Remote control of neuronal activity in transgenic mice expressing evolved G protein-coupled receptors. Neuron. 2009;63(1):27-39.

39. Hisadome K, Reimann F, Gribble FM, Trapp S. CCK stimulation of GLP-1 neurons involves $\alpha 1$-adrenoceptor-mediated increase in glutamatergic synaptic inputs. Diabetes. 2011;60(11):2701-2709.

40. Hisadome K, Reimann F, Gribble FM, Trapp S. Leptin directly depolarizes preproglucagon neurons in the nucleus tractus solitarius: electrical properties of glucagon-like Peptide 1 neurons. Diabetes. 2010;59(8):1890-1898.

41. Baggio LL, Huang Q, Brown TJ, Drucker DJ. Oxyntomodulin and glucagon-like peptide-1 differentially regulate murine food intake and energy expenditure. Gastroenterology. 2004;127(2):546-558.

42. Jessen L, et al. Suppression of food intake by glucagon-like peptide-1 receptor agonists: relative potencies and role of dipeptidyl peptidase- 4 . Endocrinology. 2012;153(12):5735-5745.

43. Katsurada K, et al. Endogenous GLP-1 acts on paraventricular nucleus to suppress feeding: projection from nucleus tractus solitarius and activation of corticotropin-releasing hormone, nesfatin-1 and oxytocin neurons. Biochem Biophys Res Commun. 2014;451(2):276-281.

44. Rinaman L, Rothe EE. GLP-1 receptor signaling contributes to anorexigenic effect of centrally administered oxytocin in rats. Am J Physiol Regul Integr Comp Physiol. 2002;283(1):R99-R106.

45. Ronveaux CC, de Lartigue G, Raybould HE. Ability of GLP-1 to decrease food intake is dependent on nutritional status. Physiol Behav. 2014;135:222-229.

46. Williams DL, Baskin DG, Schwartz MW. Leptin regulation of the anorexic response to glucagonlike peptide-1 receptor stimulation. Diabetes. 2006;55(12):3387-3393

47. Knauf C, et al. Role of central nervous system glucagon-like Peptide-1 receptors in enteric glucose sensing. Diabetes. 2008;57(10):2603-2612.

48. Knauf C, et al. Brain glucagon-like peptide-1 increases insulin secretion and muscle insulin resistance to favor hepatic glycogen storage. JClin Invest. 2005;115(12):3554-3563.

49. Lockie SH, et al. Direct control of brown adipose tissue thermogenesis by central nervous system glucagon-like peptide-1 receptor signaling. Diabetes. 2012;61(11):2753-2762.

50. Nogueiras R, et al. Direct control of peripheral lipid deposition by CNS GLP-1 receptor signaling is mediated by the sympathetic nervous system and blunted in diet-induced obesity. J Neurosci. 2009;29(18):5916-5925.

51. McMahon LR, Wellman PJ. PVN infusion of GLP1-(7-36) amide suppresses feeding but does not induce aversion or alter locomotion in rats. $\mathrm{Am} \mathrm{J}$ Physiol. 1998;274(1 Pt 2):R23-R29.

52. Williams KW, et al. Xbp1s in Pomc neurons connects ER stress with energy balance and glucose homeostasis. Cell Metab. 2014;20(3):471-482.

53. Barreto-Vianna AR, Aguila MB, Mandarimde-Lacerda CA. Effects of liraglutide in hypothalamic arcuate nucleus of obese mice. Obesity (Silver Spring). 2016;24(3):626-633.

54. Llewellyn-Smith IJ, Marina N, Manton RN, Reimann F, Gribble FM, Trapp S. Spinally projecting preproglucagon axons preferentially innervate sympathetic preganglionic neurons. Neuroscience. 2015;284:872-887.

55. Garfield AS, et al. A parabrachial-hypothalamic cholecystokinin neurocircuit controls counterregulatory responses to hypoglycemia. Cell Metab. 2014;20(6):1030-1037.

56. Verberne AJ, Sabetghadam A, Korim WS. Neural pathways that control the glucose counterregulatory response. Front Neurosci. 2014;8:38.

57. Schneeberger M, et al. Reduced $\alpha$-MSH underlies hypothalamic ER-stress-induced hepatic gluconeogenesis. Cell Rep. 2015;12(3):361-370.

58. Kooijman S, et al. Central GLP-1 receptor signalling accelerates plasma clearance of triacylglycerol and glucose by activating brown adipose tissue in mice. Diabetologia. 2015;58(11):2637-2646.

59. Coppari R, et al. The hypothalamic arcuate nucleus: a key site for mediating leptin's effects on glucose homeostasis and locomotor activity. Cell Metab. 2005;1(1):63-72.

60. Trujillo JM, Nuffer W, Ellis SL. GLP-1 receptor agonists: a review of head-to-head clinical studies. Ther Adv Endocrinol Metab. 2015;6(1):19-28.

61. Tauchi M, Zhang R, D'Alessio DA, Stern JE, Herman JP. Distribution of glucagon-like peptide-1 immunoreactivity in the hypothalamic paraventricular and supraoptic nuclei. JChem Neuroanat. 2008;36(3-4):144-149.

62. Anderberg RH, Richard JE, Hansson C, Nissbrandt H, Bergquist F, Skibicka KP. GLP-1 is both anxiogenic and antidepressant; divergent effects of acute and chronic GLP-1 on emotionality. Psychoneuroendocrinology. 2016;65:54-66.

63. Warming S, Costantino N, Court DL, Jenkins NA, Copeland NG. Simple and highly efficient BAC recombineering using galK selection. Nucleic Acids Res. 2005;33(4):e36.

64. Zhang Y, et al. Liver LXR $\alpha$ expression is crucial for whole body cholesterol homeostasis and reverse cholesterol transport in mice. JClin Invest. 2012;122(5):1688-1699.

65. Hoehn KL, et al. Insulin resistance is a cellular antioxidant defense mechanism. Proc Natl Acad Sci USA. 2009;106(42):17787-17792.

66. Zisman A, et al. Targeted disruption of the glucose transporter 4 selectively in muscle causes insulin resistance and glucose intolerance. $\mathrm{Nat}$ Med. 2000;6(8):924-928.

67. Hargus NJ, Nigam A, Bertram EH, Patel MK. Evidence for a role of Nav1.6 in facilitating increases in neuronal hyperexcitability during epileptogenesis. J Neurophysiol. 2013;110(5):1144-1157.

68. Scott MM, et al. Hcrtr1 and 2 signaling differentially regulates depression-like behaviors. Behav Brain Res. 2011;222(2):289-294.

69. Gaykema RP, et al. Characterization of excitatory and inhibitory neuron activation in the mouse medial prefrontal cortex following palatable food ingestion and food driven exploratory behavior. Front Neuroanat. 2014;8:60. 